\title{
Determinantes Fundamentales del Ratio Per: Análisis Teórico y Evidencia Empírica
}

\section{Manuel García-Ayuso Covarsí \& Juan Antonio Rueda Torres}

To cite this article: Manuel García-Ayuso Covarsí \& Juan Antonio Rueda Torres (2001) Determinantes Fundamentales del Ratio Per: Análisis Teórico y Evidencia Empírica, Spanish Journal of Finance and Accounting / Revista Española de Financiación y Contabilidad, 30:107, 129-164, DOI: $10.1080 / 02102412.2001 .10779415$

To link to this article: https://doi.org/10.1080/02102412.2001.10779415

Published online: 15 Jan 2014.

Submit your article to this journal ๘

山 Article views: 10 


\section{Manuel García- Ayuso Covarsí y Juan Antonio Rueda Torres (*) Departamento de Contabilidad y Economía Financiera. Universidad de Sevilla}

\section{DETERMINANTES} FUNDAMENTALES DEL RATIO PER: ANÁLISIS TEÓRICO Y EVIDENCIA EMPÍRICA

Resumen.-Palabras clave.-Abstract.-Key words.-1. Introducción.2. Fundamentos teóricos del empleo del ratio PER en el análisis de inversiones.-

3. Evidencia empírica publicada en relación con el comportamiento de ratio PER

y sus factores determinantes. - 4. Descripción del estudio: 4.1. Hipótesis de partida. 4.2. Muestra analizada. 4.3. Definición de las variables. 4.4. Método.-5. Resultados: 5.1. Persistencia temporal del ratio E/P.

5.2. Relación entre el ratio $\mathrm{E} / \mathrm{P}$ y el crecimiento presente y futuro de los resultados.. 5.3. Relación entre el crecimiento actual y el crecimiento futuro de los resultados. 5.4. El riesgo y el crecimiento futuro de los resultados como determinantes del E/P.-6. Conclusiones.-Bibliografía.

(*) Agradecemos al Servicio de Estudios de la Bolsa de Madrid su inestimable ayuda al hacer accesible parte de la información necesaria para la elaboración de la base de datos empleada en esta investigación. Asimismo, estamos agradecidos a Juan Carlos Gómez Sala y a Gonzalo Rubio por habernos proporcionado parte de las series de rentabilidades bursátiles necesarias para la estimación de las betas utilizadas en el análisis empírico y a los dos evaluadores anónimos de la $R E F C$ por sus comentarios y sugerencias. Este trabajo es un resultado del proyecto de investigación PB98-0415, financiado por la Dirección General de Investigación Científica y Técnica del Ministerio de Educación y Ciencia. 


\section{RESUMEN}

\section{$\mathrm{E}$} STE trabajo presenta una discusión teórica sobre los fundamentos de la utilización del ratio PER en el análisis de inversiones, así como los resultados de un análisis empírico de la persistencia del ratio, sus factores determinantes y su capacidad para explicar el crecimiento futuro de los resultados, a partir de una muestra de empresas cotizadas en la Bolsa de Madrid. La evidencia hallada indica que el ratio tiene un comportamiento persistente en el tiempo, de modo que al agrupar a las empresas en carteras, buena parte de las diferencias entre los valores correspondientes a las carteras extremas se mantienen en el tiempo. Además, encontramos que los valores extremos del ratio están asociados a importantes reversiones en el crecimiento de los resultados, lo que sugiere que el PER es un buen indicador de la existencia de componentes transitorios en el resultado. Finalmente, el análisis de los factores determinantes del ratio puso de manifiesto que ni el coeficiente beta ni el crecimiento de los resultados observado en los ejercicios futuros permiten explicar las diferencias observadas en los valores actuales del PER. Esto puede interprétarse como una muestra de la incapacidad del mercado para predecir el comportamiento futuro de los resultados, de la incapacidad de la beta para reflejar adecuadamente el riesgo asociado a los títulos, o bien de la existencia de factores determinantes de la relación resultado-precio que nada tienen que ver con el crecimiento futuro de los resultados.

\section{PALABRAS CLAVE}

Análisis fundamental; Ratio PER; Valoración; Crecimiento; Persistencia del resultado.

\section{ABSTRACT}

This paper presents a theoretical discussion on the foundation of the use of the P/E ratio in investment analysis and presents the results of an empirical analysis of its persistence, fundamental determinants and ex 
planatory ability for future earnings growth on the basis of a sample of firms listed in the Madrid Stock Exchange. We found that the P/E ratio has a high degree of time-series persistence, as when firms are grouped in portfolios, a large amount of the difference between de median values of the ratio for the extreme portfolios persists up to 10 years after formation. Our results also indicate that extreme values of the $\mathrm{P} / \mathrm{E}$ ratio are associated to significant earnings, and therefore, that the ratio is a good indicator of the existence of transitory components in earnings. Finally, the analysis if the determinants of the $\mathrm{P} / \mathrm{E}$ ratio revealed that neither the CAPM beta nor subsequent observed earnings growth account for the cross-sectional differences in the values of the ratio. This may be interpreted as a proof of the market's lack of ability to predict future earnings from historic information, of the lack of validity of beta as a proxy for risk, or of the existence of certain determinants of the earnings-price relationship that have nothing to do with future earnings growth.

\section{KEY WORDS}

Fundamental analysis; P/E ratio; Valuation; Growth; Earnings persistence.

\section{INTRODUCCIÓN}

El ratio PER (1) es la variable empleada con mayor profusión en el análisis de inversiones en los mercados de valores y, probablemente, una de las que con mayor frecuencia se interpreta erróneamente. El PER se ha utilizado tradicionalmente como consecuencia de sü sencilleź (relaciona el resultado con el precio que el mercado está dispuesto a pagar

(1) A lo largo de este trabajo emplearemos la abreviatura PER para designar al cociente entre el valor de mercado de la empresa y su resultadó contable en el ejercicio (Price-to-Earnings Ratio), ya que esa es la denominación aceptada con carácter general en la investigación y en la práctica del análisis financiero. No obstante, én el repaso de la literatura que presentamos en la sección segunda, haremos referencia al inverso del PER designándolo como ratio E/P. Además, en el análisis empírico optamos por utilizar esta última construcción, por ser menos sensible a la existencia de valores extremos o indetermidados, por lo que en la descripción del estudio y el análisis de los resultados, hablaremos generalmente del ratió $\mathrm{E} / \mathrm{P}$. 
por él), su facilidad de cálculo y, su disponibilidad. No obstante, como afirma Damodaran [1996], frecuentemente se ignora la relación existente entre el ratio PER y los determinantes fundamentales del valor de la empresa, lo que conduce a errores significativos en su empleo.

Tradicionalmente, el ratio se ha utilizado para la valoración de acciones interpretándolo como un indicador eficiente de las expectativas del mercado en relación con el riesgo asociado a la empresa y el crecimiento futuro de sus resultados. En general, se supone que los precios son fijados en el mercado mediante un mecanismo eficiente, por lo que también se asume que las expectativas se formulan de manera racional y no se desvían sistemáticamente de las realizaciones subsiguientes. Además, el empleo del ratio PER está basado en la suposición de que los resultados actuales y pasados son un buen indicador de los resultados futuros, es decir, que los componentes transitorios no constituyen una proporción importante de los resultados. De acuerdo con estos planteamientos, Beaver y Morse [1978] (en lo sucesivo B\&M), llevaron a cabo el que puede considerarse primer análisis empírico riguroso de los factores determinantes del PER, llegando a la conclusión que ni el crecimiento futuro de los resultados ni el coeficiente beta del CAPM permiten explicar las diferencias de corte transversal en el PER de las acciones.

A lo largo de las últimas dos décadas, la investigación contable ha incrementado sustancialmente nuestro conocimiento sobre el funcionamiento de los mercados de capitales y ha venido a demostrar que las suposiciones anteriores no pueden sostenerse sin reservas. Así, existe un elevado número de estudios que han documentado con precisión la existencia de anomalías en la valoración de las acciones a partir de la información públicamente disponible, lo que explicaría la existencia de distorsiones en la relación resultado-precio [Ball, 1992] debidas al hecho de que los precios reflejan expectativas erróneas del mercado en relación con la capacidad de generación de riqueza por la empresa. Esto implica que los títulos caracterizados por un ratio PER relativamente elevado (reducido) serán sobrevalorados (infravalorạdos), de manera que una estrategia de inversión contraria permitiría realizar una rentabilidad anormal significativa por término medio. Por otra parte, podemos suponer que el mercado se comporta de manera eficiente en la formación de los precios y que son los resultados contables los que introducen distorsiones en los valores del PER al no constituir una medida suficiente de la riqueza generada por la empresa. En tal caso, es posible que los valores extremadamente elevados (reducidos) del ratio PER se deban a la existencia de empresas con resultados temporalmente deprimidos (elevados). A este respecto, las conclusiones del trabajo de Lipe [1986] han hecho 
que se acepte con carácter general la idea de que los precios no reflejan los componentes transitorios del resultado contable, sino que únicamente reaccionan ante cambios en los componentes permanentes, por lo que las diferencias en el PER de las empresas no pueden interpretarse si no se toma en consideración la persistencia de sus resultados.

A la luz de estos y otros desarrollos recientes de la investigación empírica en contabilidad centrada en el mercado de capitales, este trabajo presenta una discusión teórica del significado económico y los factores determinantes del ratio PER y analiza su comportamiento en series temporales, así como su relación con el crecimiento futuro de los resultados y el riesgo medido a través de la beta del CAPM, a partir de una muestra de empresas admitidas a cotización en la Bolsa de Madrid.

La sección siguiente está dedicada a la discusión de los fundamentos teóricos subyacentes al empleo del ratio PER y de sus factores determinantes. La sección tercera ofrece un repaso de la evidencia empírica publicada hasta la fecha en relación con estos últimos. La sección cuarta contiene una descripción del estudio empírico realizado en el marco del mercado de valores español. La sección quinta presenta y analiza los resultados obtenidos $y$, finalmente, la sección sexta recoge las principales conclusiones de la investigación.

\section{FUNDAMENTOS TEÓRICOS DEL EMPLEO DEL RATIO PER EN EL ANÁLISIS DE INVERSIONES:}

En una primera aproximación al significado del ratio PER, podría asumirse que los resultados obtenidos por la empresa en cada uno de los ejercicios futuros serán iguales al resultado del ejercicio corriente, $x_{i}, \mathrm{y}$ que los resultados futuros serán íntegramente repartidos a los accionistas como dividendos. En tal caso, el precio de las acciones en el momento $t, P_{t}$, quedará determinado en el mercado como el valor actualizado de la renta perpetua de cuantía constante igual al nivel actual de resultados, $x_{t}$. De acuerdo con Modigliani y Miller [1958], el ratio PER puede expresarse entonces como:

$$
\frac{P_{t}}{x_{t}}=\frac{1}{\rho}
$$

donde $\rho$ es la tasa de rentabilidad exigida por los accionistas, que será función del riesgo percibido por el mercado en relación con el título considerado. 
La ecuación [1] es susceptible de generalización admitiendo una tasa constante $g$ de crecimiento de los resultados futuros, y suponiendo que la empresa financia todas sus inversiones reteniendo una fracción constante $q$ de sus resultados en cada ejercicio y que reparte la fracción restante $k=(1-q)$ como dividendos. Bajo tales supuestos, el ratio PER puede expresarse, de acuerdo con el modelo de Gordon y Shapiro [1956], como:

$$
\frac{P_{t}}{x_{t}}=\frac{k(1+g)}{\rho-g}
$$

La tasa de rentabilidad financiera o rentabilidad sobre los recursos propios en el ejercicio corriente puede definirse como $R O E=x_{t} / B_{t}$, donde $B_{1}$ es el valor contable de los recursos propios de la empresa al término del ejercicio corriente. Si se supone que esta tasa se mantiene constante en cada uno de los ejercicio siguientes, la tasa $g$ de crecimiento de los resultados (y de los recursos propios y dividendos) será igual a $q \cdot R O E /(1-q \cdot R O E)$, y la ecuación [2] puede expresarse entonces como:

$$
\begin{gathered}
\frac{P_{t}}{x_{t}}=\frac{1}{\rho}+\left(1+\frac{R O E-\rho}{\rho \cdot R O E}\right)\left(\frac{g}{r-g}\right)= \\
=\frac{1}{r_{F}}-\frac{\rho-r_{F}}{\rho-r_{F}}+\left(1+\frac{R O E-\rho}{\rho \cdot R O E}\right)\left(\frac{g}{r-g}\right)=F I-F R+F F \cdot G
\end{gathered}
$$

La ecuación [3], propuesta por Leibowitz y Kogelman [1990], permite descomponer las observaciones del PER en función de cuatro factores (2):

- Un factor interés, $F I=1 / r_{F}$, igual al inverso de la tasa de rentabilidad $r_{F}$ de un activo considerado libre de riesgo. Este factor representa el valor normal o bașe del ratio PER para cualquier compañía bajo el supuesto de ausencia de riesgo y de un crecimiento esperado nulo.

- Un. factor riesgo, $F R=\left(\rho-r_{F}\right) / p r_{F}$, que depende fundamentalmente de la diferencia entre tasa de rentabilidad exigida respecțo de la clase de riesgo del título en cuestión y el tipo de interés del activo considerado exento de riesgo. El valor de este factor es tanto mayor cuanto mayor sea la rentabilidad exigida, que será función del riesgo percibido por el mercado.

(2) Fernández [1999] proporcionan ejemplos riuméricos que ilustran el efecto de cada uno de estos factores sobre los valores observados del ratio PER. 
- Un factor crecimiento, $G=g /(\rho-g)$, que puede interpretarse como el valor actual de los incrementos de los recursos propios de la empresa en cada uno de los períodos futuros, expresado como porcentaje del valor contable de los recursos propios en $t$.

- Un factor franquicia, $F F=1+[(R O E-\rho) / \rho \cdot R O E]$, que expresa el incremento (reducción) del ratio PER inducida por cada unidad monetaria actualizada al momento $t$ de crecimiento futuro esperado de los recursos propios, e invertida en proyectos que permiten obtener una tasa de rentabilidad $r_{E}$ superior (inferior) al coste de capital $\rho$. Este factor franquicia tomará un valor mayor (menor) que la unidad cuando la tasa de rentabilidad esperada de las inversiones futuras es mayor (menor) que la tasa de rentabilidad exigida por los accionistas, amplificando (reduciendo) el incremento del ratio PER respecto de su valor normal que es inducido por las expectativas de crecimiento.

Así pues, la ecuación [3] sugiere que el crecimiento de la empresa no crea per se valor para los accionistas y se traduce en un ratio PER significativamente superior a su valor definido por la ecuación [1]. Ello sólo será posible si las nuevas inversiones explotan oportunidades de franquicia que permitan a la empresa obtener una rentabilidad superior a la tasa $\rho$ exigida por el mercado con respecto a su clase de riesgo. En la medida en que es de esperar que las fuerzas competitivas del mercado conduzcan a largo plazo a la desaparición de la posibilidad de explotar de forma monopolística las oportunidades de franquicia, ello se traducirá en un equilibrio entre la tasa de rentabilidad obtenida sobre los recursos invertidos en el mercado real y la tasa de rentabilidad exigida por los inversores en el mercado de capitales respecto de cada empresa (igual al coste de capital), y ello inducirá a su vez la convergencia de los valores del ratio PER con respecto al valor de equilibrio para cada nivel de riesgo (3).

(3) Damodaran [1996] presenta una discusión sobre los factores determinantes del PER, que ilustra con ejemplos en los que se estima el valor teórico del ratio PER a partir de sus factores determinantes y los compara con los valores observados para detectar posibles errores en la valoración de los títulos. 


\section{EVIDENCIA EMPÍRICA PUBLICADA EN RELACIÓN CON EL COMPORTAMIENTO DEL RATIO PER Y SUS FACTORES DETERMINANTES}

El trabajo de Beaver y Morse [1978] fue el primero en contrastar la posibilidad de que, conforme con la teoría, las diferencias en las observaciones del ratio PER puedan ser explicadas por las diferencias en las expectativas del mercado acerca del crecimiento futuro de los resultados de cada empresa y del riesgo inherente a la inversión en su capital.

La evidencia obtenida por B\&M pone de manifiesto que los títulos caracterizados por unos valores relativos (positivos) extremadamente altos y bajos del ratio PER al término de un ejercicio tienden a mantener esos mismos valores relativos en los años sucesivos, y sólo a muy largo plazo se aprecia una completa reversión de dichas observaciones. Sin embargo, la correlación entre el ratio PER y el crecimiento de los resultados únicamente parece ser significativamente positiva en los años inmediatamente siguientes al corriente, sugiriendo que las diferencias persistentes en las observaciones del ratio PER no reflejan diferencias en las expectativas de los inversores en relación con el crecimiento de los resultados de las empresas a largo plazo.

Más bien, el mercado parece valorar los resultados de cada ejercicio percibiendo su carácter permanente o transitorio, y la consiguiente reversión a muy corto plazo del crecimiento de los resultados que es atribuible a componentes de naturaleza temporal. En este sentido, B\&M constatan. que las empresas cuyo ratio PER es, extremadamente alto (bajo) en un cierto año se caracterizan' por un acentuado crecimiento negativo (positivo) de sus resultados en ese año, que revierte en los años inmediatamente siguientes. La reversión a la media de las observaciones extremas del crecimiento de los resultados fue previamente documentada, por Brooks y Buckmaster [1976], sugiriendo que un modelo de recorrido aleatorio sólo es apropiado para caracterizar el comportamiento de los resultados con respecto a las empresas que muestran una variación de sus resultados en torno al crecimiento medio observado para el mercado en su conjunto (4). Por su parte, la capacidad del ratio PER para identificar la persistencia (o transitoriedad) del crecimiento de los resultados de cada ejercicio fue puesta de manifiesto con anterioridad al trabajo de Beaver y Morse [1978] por Molodovsky [1959], siendo conocida como efecto Molodovsky.

(4) La posibilidad de que el proceso estocástico generador de los resultados se asimile a un modelo de recorrido aleatorio con carácter general fue documentada por los trabajos de Albrecht, Lookabill y McKeown [1977], Ball y Watts [1972] y Watts y Leftwich [1977]. 
Beaver, Lambert y Morse [1978] atribuyen la relación positiva entre el PER y el crecimiento de los resultados a corto plazo, al reflejo retardado en la cifra de resultados de parte de la información relevante que es previamente incorporada al precio de los títulos, de manera que los precios van por delante de los resultados (5). Sin embargo, Ou y Penman [1989] demuestran que un indicador que permite predecir el signo de la variación de los resultados en el año siguiente al corriente, utilizando para ello la información contenida en los estados financieros publicados anualmente por las empresas, muestra el mismo patrón de correlación que el ratio PER con la variación relativa de los resultados en el año corriente y en los años siguientes. Esto sugiere que tanto el PER como el indicador $\operatorname{Pr}$ (medida de naturaleza contable construida a partir de las cuentas anuales públicamente disponibles y sin necesidad de recurrir a la observación del precio de los títulos en el mercado), capturan información relevante común y permiten identificar la reversión (o persistencia) del crecimiento observado de los resultados. A su vez, Ou y Penman [1989] contrastan que la capacidad predictiva del indicador por ellos propuesto es, en todo caso, superior a la que proporciona la observación del comportamiento de los precios a lo largo del ejercicio corriente. Por tanto, una media de pronóstico de los resultados a un año basada exclusivamente en la explotación de la información contable, o en la combinación de una señal informativa contable (la cifra de resultados del ejercicio corriente) y de la señal que proporcionan los precios de los títulos en el mercado, mejora considerablemente la predicción basada exclusivamente en la información aportada por el mercado.

El hecho de que el múltiplo aplicado por el mercado para capitalizar los resultados de cada ejercicio y valorar los títulos difiera sistemáticamente de unas empresas a otras, y que no sea función del crecimiento de los resultados a largo plazo, tal y como demuestran B\&M, puede ser objeto de una triple interpretación en el marco de la hipótesis de eficiencia del mercado (6):

(5) Esta idea fue posteriormente corroborada por Kothari y Shanken [1992], al comprobar que una ampliación hacia atrás de la ventana fortalecía la asociación entre los precios de las acciones y los résultados contables en muestras de corte transversal.

(6) Alternativamente, es posible interpretar que el mercado no es eficiente, de manera que los valores extremos más altos y bajos del ratio PER estarían asociados a la existencia de títulos sobrevalorados e infravalorados por el mercado. Esta interpretación sugiere que los valores relativos del PER permiten predecir el comportamiento futuro de los precios, en lugar de los resultados futuros, lo que constituye una anomalía con respecto a la hipótesis de eficiencia contrastada originariamente por Basu [1977]. 
- En primer lugar, las diferencias en el coste de capital de las empresas (y en el riesgo percibido por los accionistas), pueden explicar que el mercado aplique una tasa de capitalización a los resultados de cada período que sea persistentemente diferente de unas empresas a otras. En este sentido, Litzenberger y Rao [1971] demuestran que el inverso del PER (el ratio $\mathrm{E} / \mathrm{P}$ ) puede caracterizarse como una función lineal del riesgo medido por el coeficiente beta del CAPM y del crecimiento futuro de los resultados. Sin embargo, $B \& M$ no obtuvieron resultados consistentes con la hipótesis de que las diferencias en el riesgo medido por la beta expliquen la variación de corte transversal del ratio PER.

- En segundo lugar, cabe la posibilidad de que las tasas de crecimiento efectivamente observadas ex post para los resultados no concuerden con las expectativas ex ante del mercado en relación con el crecimiento futuro de los resultados, siendo estas últimas las que realmente son reflejadas por los precios y, consiguientemente, por loś valores observados dèl ratio PER. Esta posibilidad es considerada por Zarowin [1990], corroborando quie las expectativas de crecimiento a largo plazo de los resultados, subrogadas por una medida de consenso de los pronósticos elaborados por los analistas financieros, muestran una relación significativamente positiva con las observaciones del ratio $\mathrm{E} / \mathrm{P}$.

- Por último, 'las diferencias en los procedimientos contables empleados por las empresás para determinar su cifra de resultados en cada ejercicio también pueden ser persistentes y reflejarse en una diferencia igualmente duradera de sus ratios PER.

En relación con la última de las posibilidades apuntadas, Penman [1996, p. 244] argumenta que el ratio PER es un «producto de la contabilidad empleada» en el cómputo de las cifras de resultados. En general, esta cifra es una medida imperfecta del valor añadido en cada período, de manera que, en un mercádo eficiente, los resultados futuros no serán proyectados exclusivamente a partir de los resultados del ejercicio 'corriente. Por tanto, es de esperar que los resultados calculados de acuerdo con diferentes procedimientos contables induzcan una variabilidad en los valores del ratio PER observados sobre muestras de corte horizontal, superior a la que se deriva de las diferencias en el coste de capital de las empresas y en el crecimiento esperado de loś resultados. De acuerdo con esta argumentación, Penman [1996] demuestra que el ratio PER puede interpretarse como la relación existente entre las expectativas de resultados futuros dado el conjunto de la información total disponible, $z_{t}$; y las 
expectativas de resultados futuros formadas exclusivamente a partir del resultado del período corriente $x_{t}$, siendo dicha relación multiplicada por una constante $\rho$ igual al valor normal del ratio PER que proporciona la expresión [1].

Esta caracterización permite diferenciar dos categorías en relación con las observaciones del ratio PER:

- Los valores del PER en torno a la mediana de la distribución de corte transversal, corresponderán a títulos con respecto a los cuales puede asumirse que $z_{t} \equiv x_{t} y$, consiguientemente, el resultado observado en el período corriente proporcionará información suficiente para proyectar los resultados futuros y valorar los títulos, consistentemente con la definición del resultado contable que propone Black [1980]. En tal caso, el PER de cada título será igual a su valor normal, y las diferencias en los valores del ratio serán únicamente función de las diferencias en el coste del capital de las empresas.

- Por su parte, las empresas que muestran valores extremos del PER en un cierto período, esto es, muy superiores o inferiores al valor considerado como normal para el conjunto del mercado, se caracterizarán por tener unos resultados actuales que contienen escasa información sobre sus resultados futuros. En particular, con relación a los títulos para los que el PER es superior (inferior) al valor normal, $\rho$, el resultado contable del período corriente será muy inferior (superior) a los resultados futuros estimados utilizando toda la información disponible.

Beaver y Dukes [1973] contrastan el efecto de las políticas contables sobre las variaciones observadas en el ratio PER, observando el valor medio de dicho ratio con relación a una muestra de empresas que adoptan un método de amortización acelerada a efectos fiscales y un método de amortización lineal a efectos contables, y con respecto a otra muestra de empresas que adoptan el método de amortización acelerada a efectos fiscales y de registro contable. Consistentemente con la hipótesis de que el mercado corrige las diferencias en los procedimientos contables de las empresas para fijar el precio de equilibrio de sus títulos, el PER de las empresas que siguen el método acelerado de amortización es, por término medio, significativamente superior al de las empresas que adoptan el criterio del efecto impositivo, siendo muy similares para ambos grupos de empresas tanto su nivel de riesgo relativo (medido por el coeficiente beta) como la tasa anual de crecimiento los resultados. 
O'Donnell $[1965,1966]$ analiza una muestra de empresas del sector eléctrico norteamericano, con respecto al cual se permitió a partir de 1.954 la posibilidad de amortizar aceleradamente los activos a efectos fiscales y elaborar los estados financieros de acuerdo con el método de amortización lineal. El grupo de empresas analizadas que adoptaron el método de amortización acelerada en aquel año, tanto a efectos fiscales como contables, se caracterizaron por un crecimiento de su cifra de resultados por acción en los años siguientes y con respecto al resultado medio revelado en el período 1949-1953, durante el cual se utilizó el método de amortización lineal. Sin embargo, ese mismo grupo de empresas evidencia una reducción de su ratio PER anual medio del período anterior a 1954 al periodo posterior a ese año y en relación con el grupo de empresas que revelan un resultado normalizado mediante la activación del mayor resultado puesto de manifiesto con la amortización acelerada (reconociendo como un pasivo los impuestos cuyyo pago se difiere en cada ejercicio). Esto sugiere que el mercado percibió como «puramente ficticio» [O'Donnell, 1965, p. 139] el incremento de los resultados atribuible al diferimiento en el pago de impuestos.

Craig, Johnson " y Joy [1987] también contrastan el papel de las diferencias en la política contable de las empresas para explicar sus distintos valores del ratio PER, extendiendo el análisis de los trabajos previos para considerar el efecto de las diferencias en otros procedimientos contables distintos dél método de amortización. En concreto, estos autores consideran las diferencias en los procedimientos utilizados para la valoración de inventarios, el registro de la depreciación y, el reconocimiento de los impuestos diferidos, por una muestra de empresas industriales en cada uno de los ejercicios del período 1970-1975, defíniendo para cada procedimiento una variable dicotómica que toma valor cero cuando el criterio adoptado puede calificarse como conservador (valoración de los inventarios según la cláusula LIFO, amortización acelerada y reconocimiento como pasivo de los impuestos diferidos) y־valor uno cuando puede calificarse como agresivo (cláusula FIFO para la valoración de inventarios, amortización lineal y ausencia de activación de los impuestos diferidos). Estas variables contables se incluyen como explicativas de las observaciones del ratio $\mathrm{E} / \mathrm{P}$; junto con otra serie de variables financieras (la tasa de crecimiento de los resultados, el coeficiente beta, la tasa de reparto de dividendos y el valor de capitalización bursátil), en una regresión estimada sobre el panel de observaciones disponibles. .:

La evidencia obtenida por Craig et al. [1987] indica que los criterios adoptados en relación con la valoración de los inventarios y la activación de los impuestos diferidos contribuyen de forma significativa a explicar 
- las diferencias en los valores del ratio E/P observados de una empresa a otra. En particular, las empresas con una política contable conservadora revelan un ratio E/P significativamente inferior (un PER superior), por término medio, al de que aquellas otras que adoptan una política agresiva respecto de la valoración de inventarios y el reconocimiento de los impuestos cuyo pago se difiere en cada período. Sin embargo, al contrario que la evidencia de los trabajos previamente referidos, la decisión relativa al método de registro de la depreciación de los activos no parece influir decisivamente en las diferencias observadas del ratio E/P. Como evidencia complementaria, Craig et al. [1987] también encuentran que la tasa de reparto de dividendos y el valor de capitalización de las empresas son las variables financieras más importantes en la determinación del ratio $\mathrm{E} / \mathrm{P}$ de cada empresa mientras que, consistentemente con la evidencia de $\mathrm{B} \& \mathrm{M}$, el crecimiento de los resultados y el nivel de riesgo no juegan un papel significativo. Con respecto al tamaño de las empresas, medido por el valor de capitalización, su relación con el ratio E/P es significativamente negativa, lo que puede obedecer a la relación positiva entre el grado de conservadurismo contable y el tamaño empresarial sugerida por Watts y Zimmerman [1978].

En resumen, los estudios de O'Donnell [1965, 1966], Beaver y Dukes [1973] y Craig et al. [1987] confirman que, como sería de esperar con arreglo a la hipótesis de eficiencia del mercado, los inversores fijan el precio de los títulos teniendo presente las diferencias existentes en los procedimientos contables de las empresas. Si dichos procedimientos pueden caracterizarse como conservadores, y se traducen en unas cifras de resultados sistemáticamente inferiores al resultado permanente esperado para cada empresa, el mercado capitalizará esas cifras empleando un múltiplo superior al aplicado a los resultados revelados por las empresas que adoptan una política contable más agresiva, que son superiores al resultado permanente esperado. De acuerdo con esto, las diferencias persistentes observadas en el PER de las empresas constituyen una muestra del comportamiento racional de los inversores que corrigen el efecto positivo o negativo inducido en la cifra de resultados de cada ejercicio por las distintas políticas contables.

En la literatura española existen algunos ejemplos dẹl interés de los investigadores por el análisis empírico del comportamiento del ratio PER, si bien es escasa la evidencia con la que contamos a ese respecto y nula la relativa a sus factores determinantes. García-Ayuso [1999] llevó a cabo un análisis de la forma funcional del ratio PER a partir de una muestra de empresas cotizadas en el mercado de valores de Madrid durante el período 1990-1994, como resultado del cual concluyó que no 
existe una relación de proporcionalidad estricta entre el resultado y los precios de las acciones. De ello se deduce que el ratio no es una especificación funcional apropiada para el análisis de la relación existente entre resultados y precios, dado que existen factores determinantes del precio de las acciones que la cifra de resultados contables no captura y, consiguientemente, el establecimiento de comparaciones entre empresas sobre la base de los valores observados del PER puede conducir a conclusiones inconsistentes.

\section{DESCRIPCIÓN DEL ESTUDIO}

\subsection{HIPÓTESIS DE PARTIDA}

En línea con la argumentación teórica expuesta y asumiendo la eficiencia del mercado, nuestro estudio pretende contrastar la hipótesis de que las diferencias existentes entre lós valores observados del ratio PER en muestras de corte transversal vienen determinadas por las diferencias en:

- El crecimiento futuro de los resultados, de manera que es de esperar una relación positiva entre las observaciones del ratio PER en un cierto año y del crecimiento de los resultados en los años siguientes. En este sentido, suponemos que las realizaciones ex-post del crecimiento coinciden, por término medio, con las expectativas ex-ante del mercado.

- El coste de capital; asumiendo la validez del CAPM como modelo representativo de equilibrio entre la rentabilidad esperada por los inversares con respecto a cada título (o el coste de capital de cada empresa) y su riesgo medido exclusivamente por el coeficiente beta, cabe esperar que exista una relación negativa entre el ratio PER y la beta.

\subsection{MUESTRA ANALIZADA}

Para contrastar la hipótesis anterior, construimos una base de datos con información correspondiente a una muestra de empresas admitidas a:negociación en la Bolșa de Madrid, para las que se encontraban disponibles los siguientes datos: 
- Las cifras de resultado ordinario correspondientes a los ejercicios cerrados entre 1982 y 1997. Para el subperíodo 1982-1989, estas cifras fueron recopiladas del Sistema de Información Bursátil (SIB) de la Sociedad Rectora de la Bolsa de Madrid. A partir del año 1990, se utilizaron las cuentas anuales depositadas por las sociedades en la Comisión Nacional del Mercado de Valores y publicadas por ésta.

- Precio de las acciones y número total de títulos admitidos a cotización al cierre de la última sesión de cada año. Estos datos fueron obtenidos de los Boletines Oficiales de Cotización de la Bolsa de Madrid.

- Rentabilidades mensuales de las acciones desde enero de 1983 hasta diciembre de 1998. La fuente primaria de la que se obtuvo esta información $\mathrm{Cue}$ una base de datos con rendimientos de 168 empresas desde enero de 1963 hasta junio de 1996. Esta base fue completada calculando las rentabilidades mensuales de los títulos que figuraban en la misma para el período comprendido entre julio de 1996 y diciembre de 1998. Asimismo, se calcularon los rendimientos de otros títulos no incluidos en la base originaria, especialmènte durante los primeros años del estudio. Para realizar estas dos tareas recurrimos a la información de precios y dividendos registrada en los Anexos mensuales de los Boletines Oficiales de Cotización de la Bolsa de Madrid.

La muestra resultante quedó integrada por 168 empresas, para las que se dispone de un total de 1.651 observaciones empresa-año a lo largo del período 1982-1997. El número de observaciones en cada uno de los años oscila entre un mínimo de 71 en el año 1982 y un máximo de 120 en 1989.

Por otra parte, a efectos de estimación de la beta de los títulos incluidos en la muestra en cada año del período 1982-1997, fue preciso disponer de sus series de rentabilidades bursátiles mensuales pasadas durante, al menos, los tres últimos años. La sub-muestra de empresas para las que pudo disponerse de rentabilidades pasadas es especialmente reducida, en relación con la muestra base, en los primeros años del estudio, siendo $48 \mathrm{el}$ número mínimo de observaciones disponibles correspondientes al año 1982.

\subsection{DEFINICIÓN DE LAS VARIABLES}

Las variables calculadas para cada empresa y año, cuyos estadísticos descriptivos figuran en la Tabla 1, son las siguientes: 
TABLA 1

ESTADÍSTICOS DESCRIPTIVOS'

\begin{tabular}{|l|r|r|r|r|}
\cline { 2 - 5 } \multicolumn{1}{c|}{} & $g_{x}$ & $E / P$ & \multicolumn{1}{c|}{$B P A$} & $\beta$ \\
\hline Media & 0,1020 & $-0,0562$ & 455,8138 & 1,0486 \\
\hline Mediana & 0,0895 & 0,0927 & 187,0508 & 1,0411 \\
\hline Desv. estándar & 7,3549 & 1,2508 & 1171,1255 & 0,4519 \\
\hline Máximo & 106,5000 & 11,9375 & 12896,1190 & 2,3469 \\
\hline Mínimo & $-194,0000$ & $-20,2067$ & $-4583,3333$ & 0,0222 \\
\hline Asimetría & $-8,9106$ & $-8,5836$ & 5,3636 & 0,1223 \\
\hline Curtosis & 373,2352 & 120,6612 & 44,3126 & $-0,5247$ \\
\hline
\end{tabular}

$1 \quad g_{x}$ es la tasa de crecimiento anual del resultado ordinario por acción; $\mathrm{E} / \mathrm{P}$ es el cociente de dividir el resultado de cada ejercicio entre el precio observado a fin de año; BPA es el resultado ordinario por acción del ejercicio cerrado en cada año; y $\beta$ es el coeficiente beta estimado para cada título por la pendiente del modelo de mercado sobre un máximo de sesenta meses (y un mínimo de treinta y seis meses) previos al término de cada año.

- Ratio E/P, definido como el cociente entre la cifra de resultado ordinario por acción del año y el precio por acción al final de la última sesión del ejercicio. El número de acciones en circulación al cierre del ejercicio, necesario para calcular el beneficio por acción, se ajustó para tener en cuenta los posibles desdobles (splits) del nominal de las acciones llevados a cabo en cada año. La utilización del ratio $\mathrm{E} / \mathrm{P}$, en lugar de su inverso (el PER), viene motivada por el deseo de reducir al mínimo las observaciones extremas que aparecen en el PER cuando el resultado toma valores próximos. Además, el empleo del E/P conduce a resultados que son comparables con los de trabajos anteriores que, generalmente, adoptaron esta construcción para el ratio [Zarowin, 1990; Penman, 1996].

- Tasa de crecimiento de los resultados en cada año, calculada como la variación de la cifra de resultados ordinarios por acción entre dos ejercicios consecutivos dividida por el resultado ordinario por acción del primer ejercicio en valor absoluto. La utilización del denominador en valor absoluto permite que el signo de la tasa de crecimiento refleje el sentido de la variación experimentada por los resultados:

$$
g x_{i \tau}=\frac{x_{i \tau}-x_{i, r-1}}{\left|x_{i, r-1}\right|}
$$


- El coeficiente beta, estimado para cada valor y año sobre un horizonte temporal de sesenta meses como máximo y de treinta y seis meses como mínimo, previo al término del año considerado, como pendiente del modelo de mercado:

$$
r_{i t}=\alpha_{i t}+\beta_{i t} r_{m t}+e_{i t} t=-1, \ldots,-60
$$

donde $r_{i t}$ es la rentabilidad del título $i$ en el mes $t$, calculada como la variación relativa del precio del término del mes $t-1$ al término del mes $t$ ajustada por el dividendo líquido pagado durante el mes y por los posibles splits (7) y rmt es la rentabilidad de la cartera de mercado, estimada como variación mensual relativa del Índice General de la Bolsa de Madrid (IGBM). Obviamente, la forma adoptada para el cálculo de la beta implica asumir la estabilidad de cada estimación durante los doce meses siguientes; sin embargo, al igual que con respecto al ratio $\mathrm{E} / \mathrm{P}$, se trató de utilizar una estimación de la beta factible al término de cada año.

TABLA 2

CORRELACIÓN ENTRE VARIABLES ANALIZADAS ${ }^{\prime}$

\begin{tabular}{|l|r|r|r|r|r|r|r|}
\cline { 2 - 8 } \multicolumn{1}{c|}{} & \multicolumn{1}{c|}{$g_{x, t}$} & $g_{x, t+1}$ & \multicolumn{1}{c|}{$g_{x, t+2}$} & \multicolumn{1}{c|}{$g_{x, t+3}$} & \multicolumn{1}{c|}{$E / P$} & \multicolumn{1}{c|}{$B P A$} & $\beta$ \\
\hline$g_{x, t}$ & 1,0000 & & & & & & \\
\hline$g_{x, t+1}$ & 0,0144 & 1,0000 & & & & & \\
\hline$g_{x, t+2}$ & $-0,0047$ & 0,0144 & 1,0000 & & & & \\
\hline$g_{x, t+3}$ & $-0,0262$ & $-0,0047$ & 0,0172 & 1,0000 & & & \\
\hline$E / P$ & 0,0669 & $-0,0046$ & $-0,0350$ & $-0,0516$ & 1,0000 & & \\
\hline$B P A$ & 0,0583 & $-0,0097$ & $-0,0309$ & $-0,0522$ & 0,2915 & 1,0000 & \\
\hline$\beta$ & 0,0264 & 0,0253 & 0,0586 & 0,0699 & $-0,1771$ & $-0,2335$ & 1,0000 \\
\hline
\end{tabular}

1 Las cifras que figuran en la tabla son los coeficientes de correlación lineal (o de Pearson) entre cada par de variables. $g_{x, t+k}$ es la tasa de crecimiento del resultado por acción correspondiente al año $t+k(k=0,1,2,3)$; $\mathrm{E} / \mathrm{P}$ es el cociente de dividir el resultado de cada ejercicio entre el precio observado a fin de año; BPA es el resultado ordinario por acción del ejercicio cerrado en cada año; y $\beta$ es el coeficiente beta estimado para cada título por la pendiente del modelo de mercado sobre un máximo de sesenta meses (y un mínimo de treinta y seis meses) previos al término de cada año.

(7) Las rentabilidades también se ajustaron por las ampliaciones de capital habidas en cada mes. Para ello, se sumó a la variación en el precio de la acción y al dividendo pagado, el precio de mercado de los derechos de suscripción preferente en el primer día de negociación de los mismos. 


\subsection{MÉtodo}

El método de estudio que seguimos se articula en tres etapas:

- Al término de cada año $t$ del período 1982-1996 los títulos disponibles en la muestra se ordenan de acuerdo con sus valores relativos del ratio E/P y se agrupan en carteras. En concreto, optamos por reunir de manera independiente las observaciones negativas del ratio $\mathrm{E} / \mathrm{P}$ y formamos diez carteras de aproximadamente igual número de títulos con las observaciones positivas del ratio (8). La composición de las carteras formádas en cada año $t$ se mantuvo en los años siguientes, de manera que las empresas incluidas en una cartera en el año $t$ se mantienen en dicha cartera en los años siguientes $t+\tau$ con independencia de cual sea su ratio $\mathrm{E} / \mathrm{P}$ en esos años. Por otra parte, hay que señalar que el número $\tau$ de años siguientes a cada año $t$ que resultan disponibles se reduce progresivamente desde un máximo de quinçe años para las carteras formadas en 1982 hasta un solo año para las carteras formadas en 1996.

- En segundo lugar, calculamos los valores médianos del ratio E/P y de la tasa de crecimiento de los resultados para cada una de las carteras en el año corriente $t \mathrm{y}$ en cada año $t+\tau$. La utilización de los valores medianos por carteras de las variables estudiadäs, en lugar de valores medios, estuvo motivada por el intento de atenuar la influencia de las observaciones extremas.

- Por último, con los valores medianos por carteras tanto del ratio $\mathrm{E} / \mathrm{P}$ como del crecimiento de lós resultádos calculamos una media para cada año $t$ de formación de las carteras y cada uno de los años siguientes $t+\tau$. De igual modo calculamos la media de los coeficientes de correlación observados entre las medianas por carteras del ratio $\mathrm{E} / \mathrm{P}$ y crecimiento de los resultados en los años siguientes al de formación de aquéllas. Finalmente, calculamos la media de los coeficientes de correlación entre las medianas por carteras del crecimiento de los resultados en el año de formación y en los años siguientes. En este sentido, con objeto de disponer de un número suficiente de observaciones para cada año $t+\tau$; el análisis del com-

(8) Decidimos no prescindir de los títulos que muestran valöres negativos del ratio E/P en cada año, como hacen otros estudios, porque su número es considerable en nuestra muestra. El análisis dé forma separada de estos títulos se justifica por la diferente interpretación que debe hacerse dèl ratio E/P cuando se computa utilizando como numerador una cifra negativa de resultados. 
portamiento del ratio $\mathrm{E} / \mathrm{P}$ y del crecimiento de los resultados de las carteras formadas en cada año $t$ se limitó a un horizonte futuro de diez años, de modo que para el año $t+10$ son disponibles seis observaciones por cartera.

Para evaluar en qué medida las diferencias observadas en los valores del ratio E/P son una consecuencia del crecimiento futuro de los resultados y el nivel de riesgo asociado a los títulos, medido por la beta del CAPM, estimamos de regresiones de corte transversal en cada uno de los años del período muestral:

$$
(E / P)_{k t}=a_{0 t}+a_{t t} \beta_{k t}+\sum_{\tau=1}^{3} b_{u} g x_{k, t+\tau}+e_{k t}
$$

donde $(\mathrm{E} / \mathrm{P})_{k t}$ es la mediana del ratio E/P para la cartera $k$ al término del año $t ; \beta_{k t}$ es la mediana del coeficiente beta de las empresas en la cartera $k$ al término del año $t ; g x_{k, t+\tau}$ es la tasa mediana de crecimiento del resultado por acción de la cartera $k$ en el año $t+\tau(\tau=1,2,3)$; y, por último, $e_{i t}$ es un término de perturbación que se asume normalmente distribuido con media cero y varianza constante.

La inclusión del coeficiente beta en la expresión [6] limitó su estimación a los títulos incluidos en la muestra para los que se dispone de la estimación de dicho parámetro. Asimismo, se suprimieron las observaciones negativas del ratio E/P, dado que es de esperar que la inclusión de estas observaciones impida observar unas relaciones significativas entre la variable dependiente y las variables independientes en [6]. Por otra parte, se optó por considerar las tasas de crecimiento de los resultados en los tres años siguientes al corriente para intentar explicar la variación de los ratios E/P en este año. La limitación a tres años del horizonte de observación de las variables explicativas estuvo motivada por la intención de disponer del mayor número posible de años para estimar la regresión [6], de manera que la extensión de ese horizonte implicaría contar con menos años al final del período muestral (9). Con el crecimiento de los resultados de los tres años siguientes al corriente, es posible estimar la ecuación [6] desde 1982 hasta 1994.

(9) Asimismo, la consideración del crecimiento en los tres años siguientes al año corriente es consistente con el modelo que es contrastado por Beaver y Morse [1978]. 


\section{RESULTADOS}

\subsection{Persistencia temporal del ratio E/P}

La Tabla 3 presenta los resultados del análisis del comportamiento en series temporales del ratio E/P. En ella se denota por E/P0 a la cartera formada por los títulos de la muestra cuyo ratio $\mathrm{E} / \mathrm{P}$ es negativo en cada año; E/P1 es la cartera que agrupa en cada año el 10 por 100 de los títulos con un ratio $\mathrm{E} / \mathrm{P}$ positivo y más reducido en términos relativos, mientras que E/P10 denota la cartera formada por el 10 por 100 de los títulos con un ratio E/P positivo y más elevado en términos relativos (10).

A la vista de la Tabla 3, resulta evidente que los valores relativos del ratio $\mathrm{E} / \mathrm{P}$ presentan una elevada persistencia temporal, ya que la ordenación de los títulos en un año cualquiera del período analizado según la distribución del ratio tiende a mantenerse en los años sucesivos. No obstante, el coeficiente de correlación entre los valores medianos del ratio $\mathrm{E} / \mathrm{P}$ de las carteras en el año $t$ de formación de las mismas y en cada uno de los años siguientes $t+\tau$ muestra una tendencia decreciente. Así, la correlación entre los rangos de las carteras según su ratio $\mathrm{E} / \mathrm{P}$ mediano en el año de formación y en el año siguiente es, por término medio, superior al 96 por 100, decreciendo la magnitud de la correlación media de forma casi monótona a medida que se consideran años sucesivamente más alejados del año de formación. Al mismo tiempo, se observa un progresivo incremento de la dispersión de las correlaciones calculadas con respecto a los años más distantes del año base, como refleja la reducción del estadístico $t$ asociado a la media de las correlaciones observadas. Sin embargo, la correlación media entre los ratios E/P medianos de las carteras en el año base y en cada uno de los diez años siguientes es positiva y superior a un 50 por 100 , tanto si se calcula la correlación lineal como si se computa la correlación de rangos. Cuando se prescinde de las observaciones negativas del ratio E/P, la persistencia de la ordenación de las carteras según su ratio E/P mediano es también evidente, si bien la magnitud de las correlaciones es menor, como reflejan las dos ứltimạs filas de la Tabla 3.

En el Gráfico 1 se representan los valores medianos del ratio E/P correspondientes a las carteras que agrupan en cada año los títulos con un ratio

(10) El número de empresas incluidas en la cartera E/P0 fluctúa ampliamente a lo largo del período muestral, oscilando entre un mínimo de 7 empresas que registraron pérdidas en 1997 ý un máximo de 28 empresas en 1992. 
TABLA 3

RATIO E/P DE LAS CARTERAS FORMADAS EN CADA AÑO $t$ SEGÚN LA DISTRIBUCIÓN DEL RATIO E/P ${ }^{1}$

\begin{tabular}{|c|c|c|c|c|c|c|c|c|c|c|c|}
\hline & $t$ & $t+1$ & $t+2$ & $t+3$ & $t+4$ & $t+5$ & $t+6$ & $t+7$ & $t+8$ & $t+9$ & $t+10$ \\
\hline $\mathrm{E} / \mathrm{P} 0$ & $-0,38737$ & $-0,22174$ & $-0,22804$ & $-0,08847$ & 0,00989 & 0,03620 & 0,04844 & 0,04696 & 0,03562 & 0,02178 & 0,01742 \\
\hline E/P 1 & 0,03381 & 0,03857 & 0,03939 & 0,03273 & 0,04677 & 0,04067 & 0,05529 & 0,07648 & 0,05861 & 0,07793 & 0,07147 \\
\hline $\mathrm{E} / \mathrm{P} 2$ & 0,06290 & 0,06639 & 0,06284 & 0,06068 & 0,05228 & 0,05706 & 0,06562 & 0,07424 & 0,07725 & 0,09284 & 0,07939 \\
\hline $\mathrm{E} / \mathrm{P} 3$ & 0,08627 & 0,08311 & 0,07360 & 0,05901 & 0,06086 & 0,06236 & 0,08316 & 0,09354 & 0,08946 & 0,07069 & 0,07911 \\
\hline $\mathrm{E} / \mathrm{P} 4$ & 0,10389 & 0,09449 & 0,08453 & 0,08318 & 0,07062 & 0,07298 & 0,03191 & 0,04902 & 0,08910 & 0,10028 & 0,09343 \\
\hline E/P 5 & 0,12220 & 0,11702 & 0,09284 & 0,08083 & 0,09113 & 0,09595 & 0,09289 & 0,09043 & 0,08631 & 0,08732 & 0,08239 \\
\hline $\mathrm{E} / \mathrm{P} 6$ & 0,15432 & 0,12898 & 0,10946 & 0,10559 & 0,09001 & 0,09991 & 0,09658 & 0,08372 & 0,09702 & 0,06932 & 0,03783 \\
\hline $\mathrm{E} / \mathrm{P} 7$ & 0,19229 & 0,16349 & 0,15316 & 0,13282 & 0,13625 & 0,12886 & 0,12005 & 0,12684 & 0,14473 & 0,10053 & 0,10571 \\
\hline $\mathrm{E} / \mathrm{P} 8$ & 0,24260 & 0,19519 & 0,16551 & 0,12431 & 0,11633 & 0,15089 & 0,15119 & 0,12485 & 0,11939 & 0,13639 & 0,16869 \\
\hline $\mathrm{E} / \mathrm{P} 9$ & 0,31066 & 0,24395 & 0,21121 & 0,18940 & 0,18385 & 0,16476 & 0,17855 & 0,16713 & 0,13169 & 0,14116 & 0,11443 \\
\hline $\mathrm{E} / \mathrm{P} 10$ & 0,48707 & 0,29939 & 0,23134 & 0,18836 & 0,16260 & 0,17099 & 0,17647 & 0,15425 & 0,16019 & 0,17017 & 0,15174 \\
\hline \multirow{4}{*}{$\begin{array}{l}\text { Pearson } \\
\text { Spearman }\end{array}$} & \multirow{4}{*}{$\therefore \quad:$} & 0,94086 & 0,86182 & 0,74239 & 0,65766 & 0,59916 & 0,55495 & 0,51863 & 0,56421 & 0,61513 & 0,58747 \\
\hline & & 16,19727 & 9,26307 & 4,90779 & 3,60479 & 2,61527 & 2,50026 & 2,77993 & 3,31343 & 3,04958 & 3,16885 \\
\hline & & 0,96303 & 0,91688 & 0,79441 & 0,74470 & 0,72149 & 0,67182 & 0,56263 & 0,57273 & 0,56104 & 0,56364 \\
\hline & & 27,16282 & 20,40140 & 6,06745 & 4,52754 & 3,36439 & 5,61149 & 2,81645 & 3,64949 & 2,68010 & 4,71105 \\
\hline$E / P>0$ & \multirow[t]{5}{*}{$\therefore \quad:$} & & & .. & $\because$ & & & & & & \\
\hline \multirow{4}{*}{$\begin{array}{l}\text { Pearson } \\
\text { Spearman }\end{array}$} & & 0,91271 & 0,85442 & 0,71127 & 0,65813 & 0,62540 & 0,60823 & 0,48148 & 0,50509 & 0,56268 & 0,50462 \\
\hline & & 8,57197 & 7,38848 & 3,69915 & 3,39467 & 2,42758 & 2,71120 & 2,23240 & 2,68887 & 2,95450 & 1,95514 \\
\hline & & 0,95071 & 0,89091 & 0,75012 & 0,72626 & 0,70689 & 0,68000 & 0,52458 & 0,53182 & 0,48398 & 0,47879 \\
\hline & & 20,11140 & 14,50060 & 4,21472 & 3,98326 & 3,23410 & 3,75356 & 2,65756 & 2,71514 & 2,49577 & 2,95072 \\
\hline
\end{tabular}

1 E/P0 es la cartera formada por las observaciones negativas del ratio E/P en cada año $t$ del período 1982-1996; E/P1 es la cartera que agrupa los títulos con un ratio E/P positivo y más reducido en cada año; y E/P10 es la cartera formada por los títulos con un ratio E/P relativamente más elevado. En cada año del período 1982-1996, y en los años siguientes disponibles, se calcula la mediana del ratio E/P para cada cartera. En las columnas figuran las medias calculadas para cada cartera y año $t+\tau(\tau=0,1, \ldots, 10)$ con todas las observaciones medianas disponibles en cada caso. Así, para el año $t$ se dispone de 15 observaciones, mientras que para el año $t+10$ sólo existen 6 observaciones. En la segunda parte de la tabla figuran las medias de los coeficientes de correlación lineal (o de Pearson) y de correlación de rangos (o de Spearman) entre el ratio E/P mediano de las carteras en el año $t$ y en cada año siguiente $t+\tau$. En la tercera parte, figuran ambos coeficientes calculados exclusivamente para las diez carteras que agrupan las observaciones positivas del ratio E/P. La cifra que aparece debajo de cada 


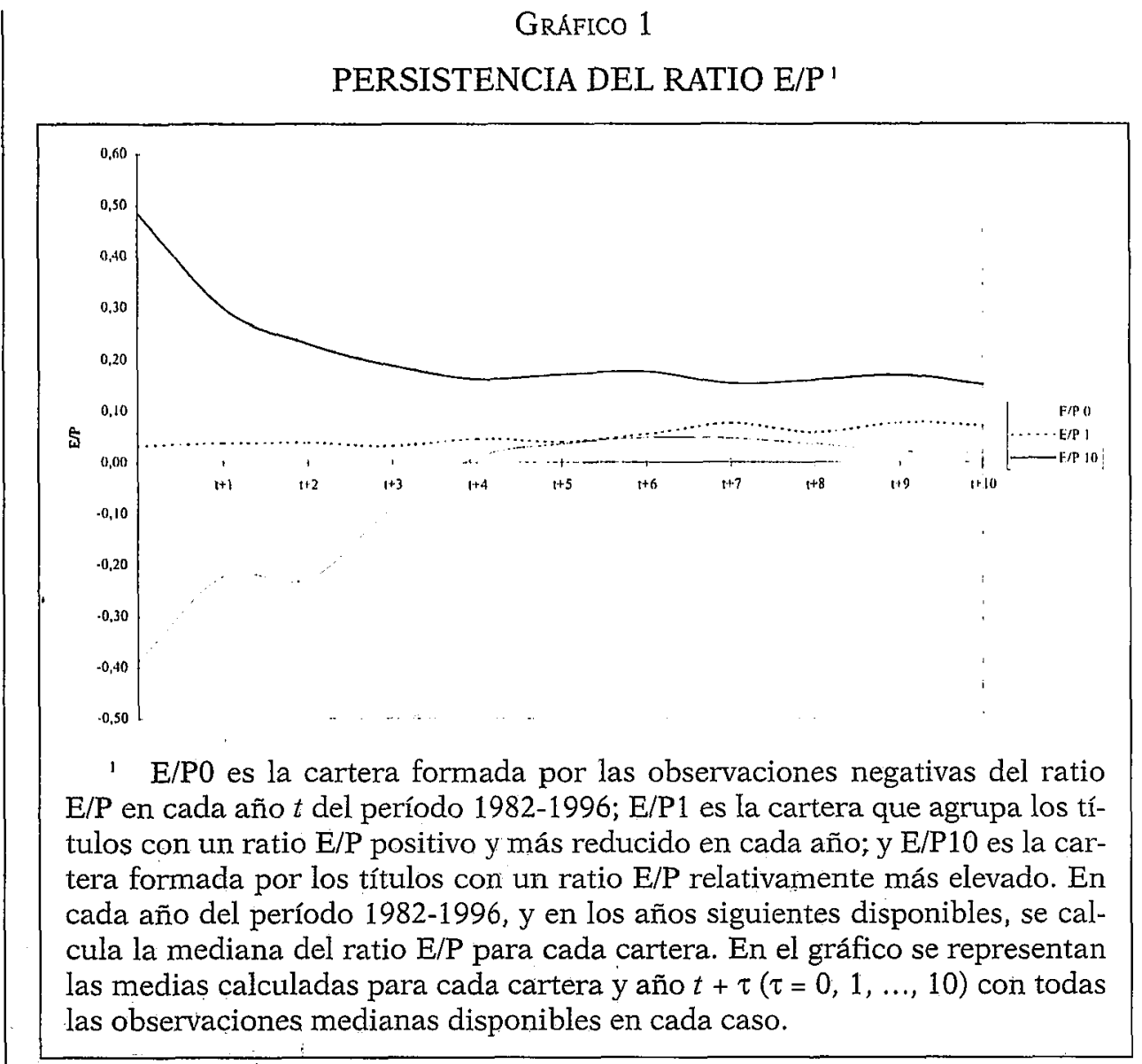

E/P negativo y con un ratio E/P positivo más alto y más bajo. Puede apreciarse cómo las empresas que muestran $\mathrm{E} / \mathrm{P}$ negativo en el año corriente continúan incurriendo en pérdidas, por término medio, hasta tres años después y, transcurridos diez años, el ratio E/P de estos títulos es el más reducido del mercado. Por su parte, las empresas con un E/P más alto en el año corriente muestran una reducción del valor del ratio durante los cuatro años siguientes que, a partir de entonces, se mantiene considerablemente estable y con un valor relativo que tiende a ser el más elevado del mercado. Las' empresas con un ratio $\mathrm{E} / \mathrm{P}$ positivo y más bajo en cada año son las que, en promedio, presentan un comportamiento más estable 
de este ratio en los años siguientes. En conjunto, el gráfico refleja una drástica atenuación a corto plazo de la diferencia entre el ratio E/P observada para las empresas con valores extremos, tanto positivos como negativo, de este ratio en cada año, si bien a largo plazo dicha diferencia tiende a persistir:

\subsection{RELACIÓN ENTRE EL RATIO E/P Y EL CRECIMIENTO PRESENTE Y FUTURO DE LOS RESULTADOS}

Para el conjunto de empresas incluidas en cada una de las once carteras identificadas al término de cada año del período 1982-1996 según la. distribución del ratio $\mathrm{E} / \mathrm{P}$, calculamos el valor mediano del crecimiento de los resultados por acción en el año de formación y en los años siguientes. A partir de estos valores medianos del crecimiento de los resultados para cada cartera y año se calculó una media para el conjunto de los años $t$ de formación de las carteras, así como para los años $t+\tau$ $(\tau=1,2, \ldots, 10)$ siguientes, tal como se refleja en la Tabla 4.

Como puede apreciarse, se produce una significativa reversión a corto plazo del crecimiento de los resultados observado para las empresas con valores relativos extremos del ratio E/P. En concreto, las empresas que muestran un E/P negativo y positivo pero muy reducido en el año corriente, tienden a experimentar una notable reducción en su cifra de resultados por acción del ejercicio corriente que, por término medio, es de un 46 por 100 para las empresas con un ratio E/P negativo y de un 10 por 100 para las empresas con un valor positivo y más reducido. Sin embargo, en el año siguiente, el resultado de estas empresas experimenta un crecimiento substancial, en torno a un 44 por 100 para las primeras y a un 61 por 100 para las segundas. Por su parte, las empresas con un ratio $\mathrm{E} / \mathrm{P}$ positivo y relativamente más alto en el año corriente, incluidas en la cartera $\mathrm{E} / \mathrm{P} 10$, son las que muestran un crecimiento de sus resultados más elevado en promedio, con la excepción de las empresas incluidas en la cartera E/P8, situándose en torno a un 21 por 100. En el año siguiente, este crecimiento se torna negativo y alcanza un nivel medio del 14 por 100 .

Para las empresas con un valor del ratio E/P próximo a la mediana del mercado en cada año, no se observa una relación consistente entre el crecimiento de los resultados en el año corriente y en los años inmediatamente siguientes. De hecho, la correlación entre el crecimiento mediano de las carteras formadas según el ratio E/P en un año y en cada uno de los años siguientes, aunque negativa por término medio, no es elevada 


\begin{tabular}{|c|c|c|c|}
\hline 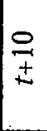 & 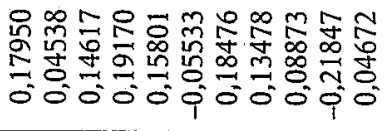 & 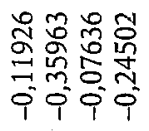 & 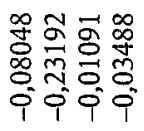 \\
\hline 主 & 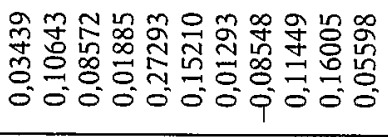 & 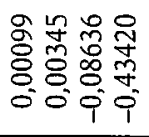 & 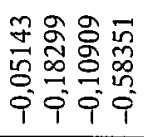 \\
\hline 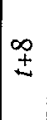 & 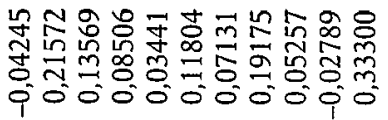 & 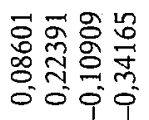 & 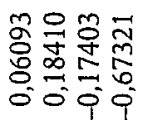 \\
\hline
\end{tabular}

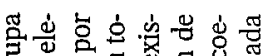

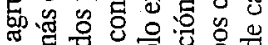

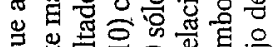

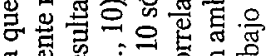

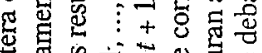
资

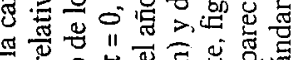
\%

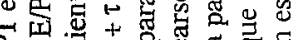
E.

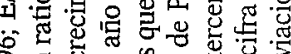

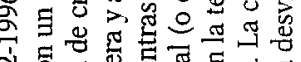

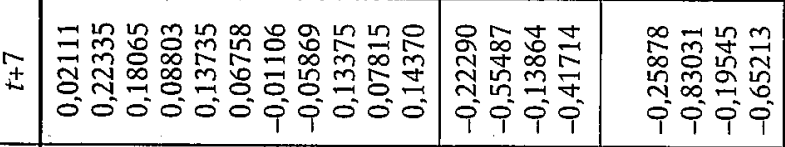

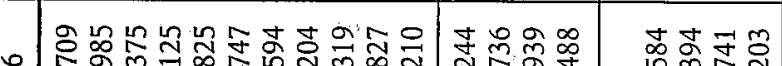

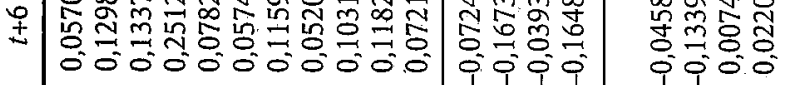

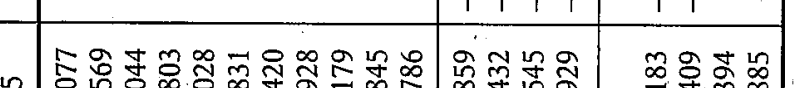

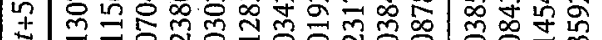

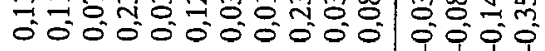
$\vec{\sim}$ 负串 iिक्ष

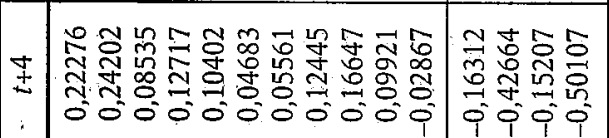
$\infty \infty$ (10

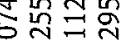
í⿴囗十

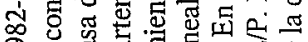

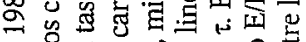

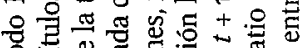
뭉

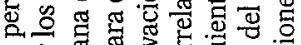

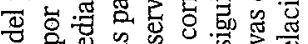

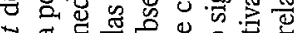

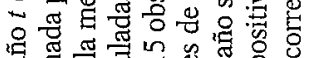

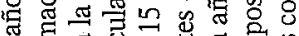

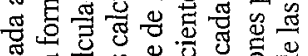
푼 둰 实 은

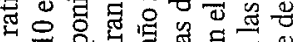
व

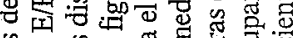

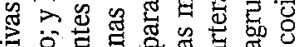

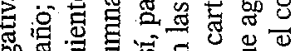

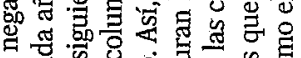
\& 正远

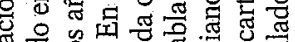

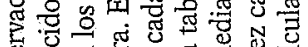

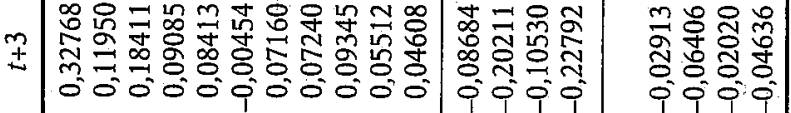

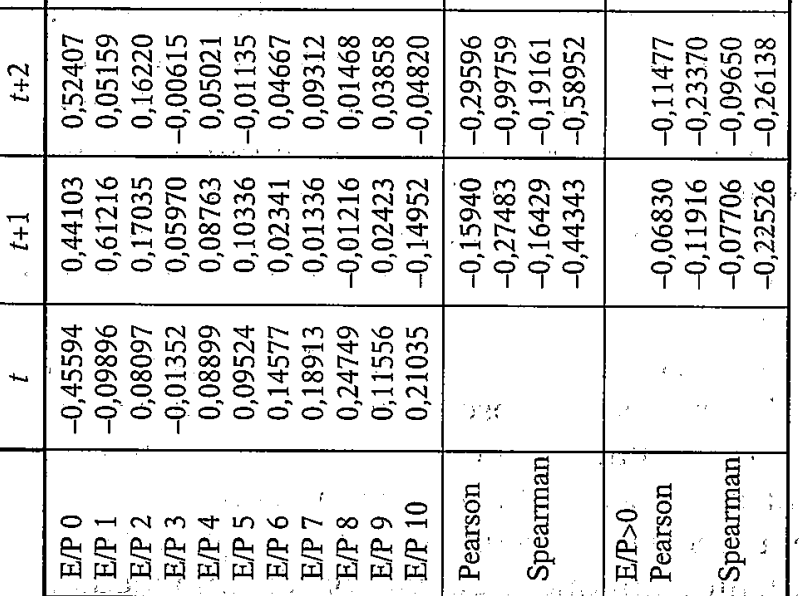

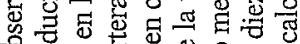

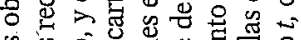

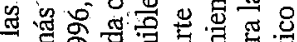

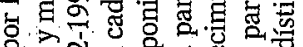

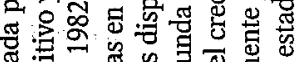

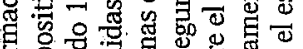

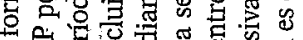

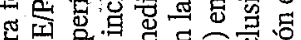

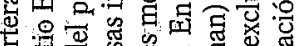

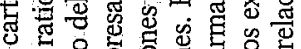

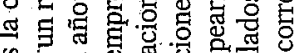

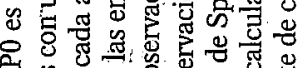

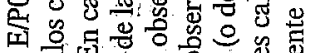

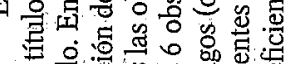

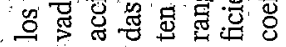


en ningún caso y fluctúa considerablemente. Esto indica que la relación existente entre el crecimiento corriente y futuro de los resultados sólo es significativa en el caso de las empresas con valores extremos del ratio $\mathrm{E} / \mathrm{P}$, para las que se observa una reversión notable, que no tiende a persistir en períodos posteriores.

La reversión en el crecimiento de los resultados de las empresas que muestran unos valores negativos y positivos más altos y más bajos del ratio E/P es especialmente evidente a la vista del Gráfico 2. Como puede apreciarse, la reversión de la tasa mediana de crecimiento de los resultados observada para las empresas con valores extremos del ratio $\mathrm{E} / \mathrm{P}$ en el

GRÁFICO 2

\section{CRECIMIENTO DE LOS RESULTADOS DE LAS CARTERAS FORMADAS SEGÚN EL RATIO E/P ${ }^{1}$}

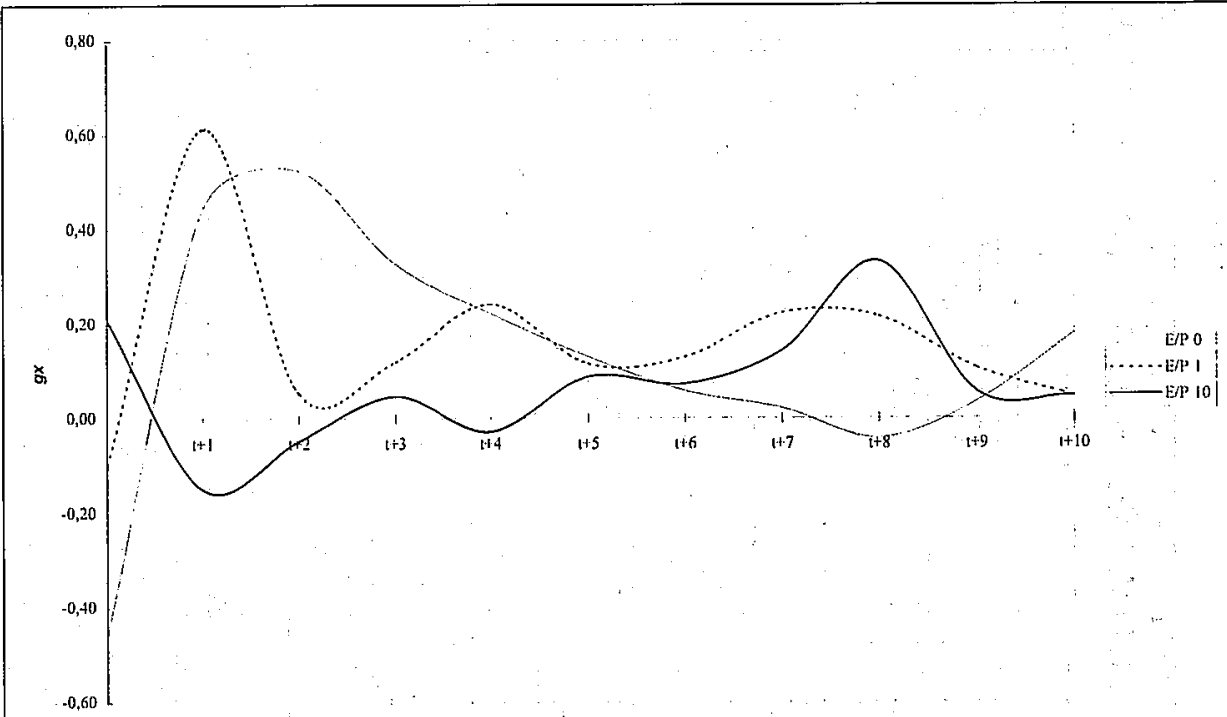

1 E/P0 es la cartera formada por las observaciones negativas del ratio E/P en cada año $t$ del período 1982-1996; E/P1 es la cartera que agrupa los títulos con un ratio E/P positivo y más reducido en cada año; y E/P10 es la cartera formada por los títulos con un ratio E/P relativamente más elevado. En cada año del período 1982-1996, y en los años siguientes disponibles, se calcula la mediana de la tasa de crecimiento de los resultados por acción de las empresas incluidas en cada cartera. En el gráfico se representan las medias calculadas para cada cartera y año $t+\tau(\tau=0,1, \ldots, 10)$ con todas las observaciones medianas disponibles en cada caso. 
RATIO E/P EN EL AÑO $t$ Y CRECIMIENTO FUTURO DE LOS RESULTADOS DE LAS CARTERAS EN CADA AÑO $t$ SEGÚN LA DISTRIBUCIÓN DEL RATIO E/P ${ }^{1}$

\begin{tabular}{|c|c|c|c|c|c|c|c|c|c|c|c|}
\hline & $\because$ & $\therefore t+1$ & $t+2$ & $t+3$ & $t+4$ & $t+5$ & $t+6$ & $t+7$ & $t+8$ & $t \div 9$ & $t+10$ \\
\hline $\mathrm{E} / \mathrm{P} 0$ & $-0,36844$ & 0,44103 & 0,52407 & 0,32768 & 276 & 077 & 09 & 0,0 & $-0,04245$ & 0,03439 & 0,17950 \\
\hline $\mathrm{E} / \mathrm{P} 1$ & 0,03305 & 0,61216 & 0,05159 & 0,11950 & 0,24202 & 0,11569 & 0,12985 & 0,22335 & 0,21572 & 0,10643 & 0,04538 \\
\hline $\mathrm{E} / \mathrm{P} 2$ & 0,06152 & 0,17035 & 0,16220 & 0,18411 & 0,08535 & 0,07044 & 0,13375 & 0,18065 & 0,13569 & 8572 & 0,14617 \\
\hline $\mathrm{E} / \mathrm{P} 3$ & 0,08432 & 0,05970 & $-0,00615$ & 0,09085 & 0,12717 & 0,23803 & 0,25125 & 0,08803 & 0,08506 & 0,01885 & 0,19170 \\
\hline $\mathrm{E} / \mathrm{P} 4$ & 0,10124 & 0,08763 & 0,05021 & 0,08413 & 0,10402 & 0,03028 & 0,07825 & 0,13735 & 0,03441 & 0,27293 & 0,15801 \\
\hline $\mathrm{E} / \mathrm{P} 5$ & 0,11920 & 0,10336 & $-0,01135$ & $-0,00454$ & 583 & 0,12831 & 747 & 58 & 0,11804 & 0,15210 & $-0,05533$ \\
\hline $\mathrm{E} / \mathrm{P} 6$ & 0,15000 & 0,02341 & 0,04667 & 0,07160 & 561 & 0,03420 & 0,11594 & $-0,01106$ & 0,07131 & 0,01293 & 0,18476 \\
\hline $\mathrm{E} / \mathrm{P} 7$ & 0,18608 & 0,01336 & 0,09312 & 0,07240 & 0,12445 & 0,01928 & 0,05204 & $-0,05869$ & 0,19175 & $-0,08548$ & 0,13478 \\
\hline $\mathrm{E} / \mathrm{P} 8$ & 0,23412 & $-0,01216$ & 0,01468 & 0,09345 & 0,16647 & 0,23179 & 0,10319 & 0,13375 & 0,05257 & 0,11449 & 0,08873 \\
\hline $\mathrm{E} / \mathrm{P} 9$ & $0 ; 29859$ & 0,02423 & 0,03858 & 0,05512 & 0,09921 & 0,03845 & 0,11827 & 0,07815 & $-0,02789$ & 005 & $-0 ; 21847$ \\
\hline $\mathrm{E} / \mathrm{P} \cdot 10$ & 0,46531 & $-0,14952$ & $-0,04820$ & 0,04608 & $-0,0$ & 0,08786 & 0,0 & 0,14370 & 300 & 598 & $0 ; 04672$ \\
\hline \multirow{4}{*}{$\begin{array}{l}\text { Pearson } \\
\text { Spearman }\end{array}$} & & & & & & 80 & & & 714 & & $-0,23313$ \\
\hline & 0,97018 & $-1,33778$ & $-1,58460$ & $-0,74648$ & $-0,50592$ & 0,03214 & $-0,11748$ & -0 & 0,39019 & 087 & $-0,81531$ \\
\hline & 0,96636 & $-0,46121$ & $-0,32078$ & $-0,26224$ & $-0,20530$ & $-0,12810$ & $-0,01091$ & 434 & 0,01932 & $-0,0$ & $-0,20303$ \\
\hline & 9,29205 & $-1,47259$ & $-1,02345$ & $-0,67121$ & -0, & 774 & 20 & $-0,11178$ & 214 & $-0,1$ & $-0,56913$ \\
\hline \multirow{3}{*}{$\begin{array}{l}\mathrm{E} / \mathrm{P}>0 \\
\text { Pearson }\end{array}$} & & & & & & & & & & & \\
\hline & 0,32810 & $=0,27694$ & 0,01635 & 0,02847 & 0,05 & 0,12915 & 102 & 0,2 & $-0,06219$ & 93 & 0,00323 \\
\hline & 0,72735 & $-0,73212$ & 0,04622 & 0,08835 & 0,16215 & 0,34028 & 0,26863 & 0,60174 & $-0,18025$ & 0,27899 & 0,00953 \\
\hline \multirow{2}{*}{$\begin{array}{l}\text { Spearman } \\
1,14338\end{array}$} & 0,44162 & $-0,34707$ & $-0,15931$ & $-0,13380$ & $-0,18586$ & $-0,08871$ & 0,01697 & $-0,08687$ & $-0,09242$ & $-0,09784$ & $-0,18788$ \\
\hline & & $-0,88078$ & $-0,42556$ & $-0,27809$ & $-0,54542$ & $-0,20458$ & 0,04919 & $-0,37851$ & $-0,33619$ & $-0,20219$ & $-0,62330$ \\
\hline
\end{tabular}

1 E/P0 es.la cartera formada por las observaciones negativas del ratio E/P en cada año $t$ del período 1982-1997; E/P1 es la cartera que agrupa los títulos con un ratio-E/P positivo y más reducido en cada año; y E/P10 es la cartera formada por los títulos con un ratio E/P relativamente más elevado. En cada año del período 1982-199.7 se calcula la mediana del ratio E/P de cada cartera, y en los años siguientes disponibles se calcula el crecimiento mediano de los resultados por acción correspondientes a las empresas incluidas en cada cartera. En la primera columna figura el ratio E/P medio de cada cartera en el año t. mientras que en las restantes columnas figura el crecimiento medio para cada cartera y año $t+\tau(\tau=1, \ldots, 10)$, calculadas con todas las observaciones medianas disponibles en cada caso. Así, para el año $t$ se dispone de 16 observaciones, mientras que para el año $t+10$ sólo existen 6 observaciones. En la segunda parte de la tabla figuran las medias de los coeficientes de correlación lineal (o de Pearson) y de correlación de rangos (o de Spearman) entre el ratio E/P mediano de las carteras en el año $t$ y el crecimiento mediano en el año $t$ y en cada año siguiente $t+\tau$. En la tercera parte, figuran ambos coeficientes calculados exclusivamente para las diez carteras que agrupan las observaciones positivas del ratio E/P. La cifra oue anarece debaio de cada coeficiente de correla 
año corriente tiene lugar en el año siguiente, mientras que a partir de este año no se observa un patrón consistente en el comportamiento del crecimiento de sus resultados. Por tanto, cuando las empresas se clasifican en carteras según sus valores relativos del ratio $\mathrm{E} / \mathrm{P}$, no parece que por término medio exista una relación persistente entre el crecimiento de los resultados en el año corriente y en períodos futuros.

Esta posibilidad se analiza específicamente en la Tabla 5, en la que aparecen las correlaciones existentes entre las observaciones medianas del ratio $\mathrm{E} / \mathrm{P}$ de las carteras formadas en un año y del crecimiento de los resultados de dichas carteras en ese año y en cada uno de los años siguientes (11). Puede apreciarse que la correlación entre el ratio E/P y el crecimiento de los resultados es positiva en el año de formación de las carteras, mientras que la correlación entre el ratio $\mathrm{E} / \mathrm{P}$ observado en el año base y el crecimiento de los resultados que se observa en los años siguientes es negativa. Sin embargo, también se aprecia que la magnitud de la correlación negativa entre el ratio E/P y el crecimiento futuro de los resultados tiende a decrecer de forma casi monótona a medida que se consideran años más distantes, al tiempo que se incrementa la desviación estándar de las correlaciones observadas, como refleja la reducción experimentada por el estadístico $t$ asociado a la correlación media. Esto confirma la ausencia de una relación significativa entre el ratio E/P y el crecimiento futuro de los resultados, de manera que el ratio y, más concretamente, sus valores extremos, sólo parecen predecir el comportamiento de los resultados a muy corto plazo. Esto es consistente con la evidencia obtenida por B\&M (12).

\subsection{RELACIÓN ENTRE EL CRECIMIENTO ACTUAL Y EL CRECIMIENTO FUTURO DE LOS RESULTADOS}

Para analizar en qué medida el crecimiento actual de los resultados permite predecir el crecimiento futuro de los resultados, agrupamos a las

(11) A diferencia de la Tabla 3, en la elaboración de la Tabla 5 se consideraron las carteras formadas en el año 1997, para las que se dispone del crecimiento de los resultados en dicho año. Así, en la primera columna de la Tabla 5 figuran las medias calculadas con los valores medianos del ratio $\mathrm{E} / \mathrm{P}$ observados para cada una de las carteras formadas de 1982 a 1997, así como la correlación media entre los valores medianos del ratio E/P y del crecimiento de los resultados de esas carteras en esos dieciséis años.

(12) En concreto, Beaver y Morse [1978] utilizan el ratio P/E, encontrando una relación positiva de este ratio con el crecimiento del año corriente, y una relación negativa y decreciente en valor absoluto con el crecimiento observado en los años siguientes. 
empresas en carteras atendiendo a la tasa de crecimiento de sus resultados por acción en cada año, analizando la correlación de las observaciones medianas de dicha variable para el conjunto de las carteras en el año de formación y en cada uno de los años siguientes. El propósito de este análisis es evaluar si el crecimiento futuro del resultado puede predecirse con mayor precisión a partir del crecimiento observado en el año corriente, obtenido exclusivamente mediante la comparación de los estados contables de una empresa en dos ejercicios consecutivos, que a partir de los valores del ratio $\mathrm{E} / \mathrm{P}$, en el que se combina la cifra de resultado de la empresa con el valor asignado por el mercado a sus acciones.

En este sentido, la Tabla 6 pone de manifiesto que las empresas que experimentan una reducción de sus resultados más acusada en cada año, incluidas en la cartera denotada como $g_{\mathrm{r}} 1$, muestran un crecimiento positivo en los años siguientes. Por su parte, las firmas con un mayor crecimiento de sus resultados, incluidas en la cartera $g_{x} 10$, ven atenuado este crecimiento en los años siguientes, si bien éste no llega a ser negativo. No obstante, el análisis de la correlación entre los rangos de las carteras según el crecimiento mediano de los resultados en el año de formación y en cada uno de los años siguientes, revela que la relación observada es, en promedio, positiva con referencia al año $t+1 \mathrm{y}$, a partir de este año, se alternan los coeficientes de correlación positivos y negativos. Esto indica que la relación entre el crecimiento corriente y futuro de los resultados es muy débil.

En conjunto, los valores relativos del ratio E/P muestran una correlación negativa con el crecimiento futuro de los resultados que parece más elevada que la encontrada al considerar los valores relativos del crecimiento observado en el año corriente. Esto sugiere que el mercado refleja en el precio de las acciones sus expectativas acerca del comportamiento de los resultados en el futuro inmediato, anticipando correctamente la reversión de las observaciones extremas del crecimiento a corto plazo de los resultados.

Los resultados del análisis dél comportamiento observado del ratio $\mathrm{E} / \mathrm{P}$ y del crecimiento de los resultados para las empresas que muestran unos valores extremos del ratio, pueden sintetizarse en los siguientes puntos:

- Por una parte, las expectativas de reversión del crecimiento de los resultados anormalmente elevado y reducido que se observa en cada ejercicio, pueden explicar en parte las diferencias entre las observaciones del ratio E/P. En concreto, las acciones de las empresas que revelan en el ejercicio corriente un resultado notablemente su- 
TABLA 6

CRECIMIENTO ANUAL DE LOS RESULTADOS DE LAS CARTERAS FORMADAS EN CADA AÑO $t$ SEGÚN LA DISTRIBUCIÓN DEL RENDIMIENTO DE LOS RESULTADOS ${ }^{1}$

\begin{tabular}{|l|r|r|r|r|r|r|r|r|r|r|r|}
\cline { 2 - 9 } \multicolumn{1}{c|}{} & $t$ & $t+1$ & $t+2$ & $t+3$ & $t+4$ & $t+5$ & $t+6$ & $t+7$ & $t+8$ & $t+9$ & $t+10$ \\
\hline$g_{x} 1$ & $-2,24586$ & 0,12906 & 0,42003 & 0,27009 & 0,04528 & $-0,19955$ & 0,89960 & 0,09769 & 0,20748 & 0,31086 & 0,55637 \\
$g_{x} 2$ & $-0,55644$ & 0,00447 & 0,04107 & 0,22773 & 0,08921 & 0,11118 & 0,14790 & 0,36296 & 0,19123 & 0,00697 & 0,27060 \\
$g_{x} 3$ & $-0,25891$ & 0,07570 & 0,03097 & 0,22015 & 0,19213 & $-0,11651$ & 0,32699 & 0,13216 & $-0,01788$ & 0,29590 & 0,22451 \\
$g_{x} 4$ & $-0,08177$ & 0,04118 & 0,03203 & 0,09157 & 0,21240 & 0,03253 & 0,01398 & 0,08429 & 0,05800 & $-0,02393$ & 0,01557 \\
$g_{x} 5$ & 0,03361 & 0,08629 & 0,07830 & 0,07873 & $-0,03395$ & 0,28808 & 0,12092 & 0,09086 & 0,16627 & 0,27223 & $-0,04097$ \\
$g_{x} 6$ & 0,14038 & 0,11728 & 0,12056 & 0,11310 & 0,13151 & 0,02602 & 0,10130 & 0,07474 & 0,07776 & 0,05007 & 0,02427 \\
$g_{x} 7$ & 0,26752 & 0,11032 & 0,11476 & 0,07827 & 0,16503 & 0,17429 & 0,12634 & 0,08717 & 0,01632 & $-0,15373$ & 0,19022 \\
$g_{x} 8$ & 0,44618 & 0,13817 & 0,06261 & 0,06542 & 0,01687 & 0,07400 & 0,11320 & 0,05657 & 0,05164 & 0,13182 & 0,34388 \\
$g_{x} 9$ & 0,82153 & 0,24112 & 0,15817 & 0,07074 & 0,08012 & $-0,02794$ & 0,01766 & 0,00765 & 0,27247 & 0,13120 & 0,22026 \\
$g_{x} 10$ & 2,37225 & 0,02604 & 0,01487 & 0,06586 & 0,09418 & 0,18170 & $-0,08736$ & $-0,17932$ & 0,36375 & 0,34912 & 0,25120 \\
\hline Pearson & & $-0,17739$ & $-0,31002$ & $-0,21149$ & $-0,14760$ & 0,13194 & $-0,17317$ & $-0,29915$ & 0,17987 & 0,08849 & $-0,17100$ \\
& & $-0,36434$ & $-0,87519$ & $-0,52520$ & $-0,36795$ & 0,28567 & $-0,39042$ & $-0,76872$ & 0,70441 & 0,20138 & $-0,33687$ \\
Spearman & & 0,01212 & $-0,07692$ & $-0,17879$ & $-0,11185$ & 0,01939 & $-0,20135$ & $-0,25000$ & 0,07879 & 0,04040 & 0,13939 \\
& & 0,02699 & $-0,22426$ & $-0,55641$ & $-0,22451$ & 0,04641 & $-0,76531$ & $-0,70929$ & 0,51334 & 0,12239 & 0,44182 \\
\hline
\end{tabular}

$g_{x} 1$ es la cartera formada por las empresas con un crecimiento relativamente más reducido de sus resultados por acción en cada año $t$ del período 1983-1996; mientras que $g_{:} 10$ es la cartera que agrupa las empresas que experimentan un mayor crecimiento. En cada año del período 1983-1996, y en los años siguientes disponibles, se calcula la mediana del crecimiento para cada cartera. En las columnas figuran las medias calculadas para cada cartera y año $t+\tau(\tau=0,1, \ldots, 10)$ con todas las observaciones medianas disponibles en cada caso. Así, para el año $t$ se dispone de 15 observaciones, mientras que para el año $t+10$ sólo existen 6 observaciones. En la segunda parte de la tabla figuran las medias de los coeficientes de correlación lineal (o de Pearson) y de correlación de rangos (o de Spearman) entre el crecimiento mediano de las carteras en el año $t$ y en cada año $t+\tau$. La cifra que aparece debajo de cada coeficiente de correlación es el estadístico $t$, calculado como el 
perior al resultado del ejercicio precedente, son valoradas a un precio relativamente reducido en comparación con la cifra de resultados por acción, y viceversa. Esto puede obedecer a que el mercado identifica el carácter transitorio de ese crecimiento y predice adecuadamente su reversión en los ejercicios siguientes. Consistentemente con esta hipótesis las acciones que muestran un ratio E/P positivo y más elevado se caracterizan por un decrecimiento del valor de este ratio en los ejercicios inmediatamente siguientes, observándose también un crecimiento de las observaciones negativas del ratio E/P. No obstante, en el caso de las acciones con un ratio E/P positivo y más reducido no se observa un patrón de crecimiento de este ratio en los años posteriores.

- Por otra parte, a pesar de la notable reversión que experimentan las observaciones extremas positivas y negativas del ratio E/P en los ejercicios inmediatamente siguientes, las diferencias entre estas observaciones persisten considerablemente a largo plazo. Paralelamente, la relación entre las observaciones del ratio $\mathrm{E} / \mathrm{P}$ y del crecimiento anual de los resultados tiende a atenuarse a medida que se consideran años sucesivamente más alejados del corriente. Consiguientemente, no parece que la persistencia de las diferencias entre las observaciones del ratio E/P obedezcan a la existencia de diferencias significativas en las expectativas del mercado acerca del crecimiento a largo plazo de los resultados de las empresas que muestran unos valores extremos de aquel ratio. En su lugar, la persistencia en las diferencias observadas del ratio $\mathrm{E} / \mathrm{P}$ puede atribuirse a dos factores:

- En primer lugar, la existencia de diferencias persistentes en la política contable de las empresas que muestran un ratio $\mathrm{E} / \mathrm{P}$ más elevado y reducido. En concreto, esta hipótesis sugiere que las empresas que se caracterizan por una política contable conservadora revelarán una cifra de resultados sesgada a la baja, mientras que las políticas más agresivas se traducirán en una cifra de resultados sesgada al alza. Si el mercado se comporta de forma eficiente, identificará estos sesgos y valorará las acciones de dichas empresas homogeneizando sus procedimientos de determinación del resultado.

- En segundo lugar, y también consistentemente con la hipótesis de eficiencia, es posible que las empresas que muestran un ratio $\mathrm{E} / \mathrm{P}$ persistentemente más alto y más bajo se caractericen por la exigencia de una rentabilidad relativamente elevada y reducida, respectivamente, por parte de los inversores. Si el CAPM es válido, 
esto implica suponer una relación positiva entre el ratio E/P y el coeficiente beta.

\subsection{EL RIESGO Y EL CRECIMIENTO FUTURO DE LOS RESULTADOS COMO DETERMINANTES DEL E/P}

Con el fin de evaluar en qué medida las variaciones observadas en los valores del ratio $\mathrm{E} / \mathrm{P}$ vienen determinadas por las diferencias en el crecimiento futuro de los resultados y al riesgo asociado a los títulos medido por medio del coeficiente beta, estimamos una regresión de los valores medianos del ratio $\mathrm{E} / \mathrm{P}$ de cada cartera sobre la mediana de las betas asociadas a las acciones incluidas en ellas y la mediana del crecimiento observado en los resultados en los tres años siguientes al de formación de las carteras. Los resultados de este análisis, contenidos en la Tabla 7, ponen claramente de manifiesto que salvo en tres de los trece años considerados, el modelo no permite explicar una proporción significativa de la varianza del ratio $\mathrm{E} / \mathrm{P}$. El coeficiente asociado a la beta sólo parece ser significativamente distinto de cero en dos años y la reducida capacidad explicativa del crecimiento de los resultados se manifiesta únicamente en los dos años siguientes al de formación de las carteras, siendo generalmente menores los valores del estadístico $t$ asociados a los coeficientes significativamente distintos de cero, a medida que se aleja el momento de medición del crecimiento en el resultado.

Con el fin de contrastar en qué medida las diferentes políticas de amortización seguidas por las empresas pueden explicar las diferencias observadas en los valores del PER, repetimos el análisis anterior considerando en el numerador del ratio la cifra de beneficios antes de amortizaciones (13). Los resultados no difieren significativamente de los anteriores, por lo que entendemos que la conclusión de $B \& M$ en relación con la posibilidad de que las diferencias en las políticas contables de las empresas expliquen las diferencias observadas en el PER, puede no ser sostenible sin reservas y, consiguientemente, debe ser tomada con cautela (al menos, en el contexto español) (14).

(13) Ni el análisis de regresión llevado a cabo sobre la base de las observaciones individuales correspondientes a las empresas incluidas en la muestra ni el realizado considerando en el numerador el beneficio antes de amortizaciones, arrojaron resultados significativamente diferentes. Estos resultados están en poder de los autores y a disposición de quienes los requieran.

(14) Obviamente, a pesar de su notable importancia, las diferencias en la amortización no son la única manifestación posible del diferente grado de conservadurismo que 


\section{TABLA 7}

\section{REGRESIÓN DEL RATIO E/P DE LAS CARTERAS FORMADAS EN CADA AÑO SOBRE LA BETA Y EL CRECIMIENTO FUTURO DE LOS RESULTADOS ${ }^{\prime}$}

\begin{tabular}{|c|c|c|c|c|c|c|c|c|}
\hline Año & $a_{0}$ & $a_{1}$ & $b_{1}$ & $b_{2}$ & $b_{3}$ & $R^{2}$ & $R^{2}$ ajustado & $F$ \\
\hline 1982 & $0,39193^{* * *}$ & $-0,01871$ & $-0,30340^{*}$ & $-0,71878^{* *}$ & $0,36551^{* *}$ & 0,88876 & 0,79976 & $9,98660^{* * *}$ \\
& 2,20426 & $-0,10161$ & $-5,47505$ & $-5,15893$ & 2,97756 & & & \\
\hline 1983 & 0,89854 & $-0,59597$ & $-0,40145$ & 0,42613 & $-0,09824$ & 0,18170 & 0,00000 & 0,27756 \\
& 1,30470 & $-0,86261$ & $-0,66493$ & 0,55415 & $-0,15817$ & & & \\
\hline 1984 & $-0,00643$ & 0,12934 & $-0,19759$ & 0,35239 & 0,12589 & 0,15477 & 0,00000 & 0,22889 \\
& $-0,01741$ & 0,41474 & $-0,49057$ & 0,80153 & 0,56849 & & & \\
\hline 1985 & 0,44978 & $-0,24290$ & 0,20221 & 0,03469 & $-0,20193$ & 0,21501 & 0,00000 & 0,34238 \\
& 1,05332 & $-0,66237$ & 0,39283 & 0,17563 & $-0,41156$ & & & \\
\hline 1986 & 0,09316 & 0,05033 & 0,01982 & $-0,29963$ & $-0,12448$ & 0,47276 & 0,05096 & 1,12082 \\
& 0,53961 & 0,27349 & 0,16130 & $-1,99575$ & $-0,77057$ & & & \\
\hline 1987 & 0,79600 & $-0,67484$ & $-0,05842$ & $-0,54833$ & $-0,03280$ & 0,55959 & 0,20726 & 1,58824 \\
& 2,38808 & $-2,02679$ & $-1,99805$ & $-1,39883$ & $-0,30200$ & & & \\
\hline 1988 & 0,28296 & $-0,17003$ & $-0,19286$ & 0,05660 & $-0,01915$ & 0,17655 & 0,00000 & 0,26800 \\
& 0,94433 & $-0,56214$ & $-0,51694$ & 0,46359 & $-0,11541$ & & & \\
\hline 1989 & 0,23367 & $-0,01542$ & $-0,35537$ & 0,08473 & 0,25362 & 0,62002 & 0,31603 & 2,03961 \\
& 0,82916 & $-0,04573$ & $-1,27761$ & 0,36402 & 0,97038 & & & \\
\hline 1990 & $0,91605^{* * *}$ & $-0,67858^{* *}$ & $-0,16613$ & $0,40080^{* * *}$ & 0,08635 & 0,84498 & 0,72097 & $6,81358^{* * *}$ \\
& 3,81522 & $-2,93079$ & $-2,38339$ & 3,60517 & 1,60632 & & & \\
\hline 1991 & 0,47548 & $-0,24110$ & 0,47407 & 0,23277 & $-0,03602$ & 0,68706 & 0,43671 & 2,74440 \\
& 1,35523 & $-0,67818$ & 1,76451 & 1,23175 & $-0,37817$ & & & \\
\hline 1992 & 0,27569 & $-0,08278$ & $-0,06547$ & $-0,40840^{* * *}$ & 0,11617 & 0,83889 & 0,71000 & $6,50855^{* * *}$ \\
& 2,43976 & $-0,74555$ & $-1,15370$ & $-3,23244$ & 0,42639 & & & \\
\hline 1993 & 0,01580 & 0,01601 & $-0,15451$ & 0,76656 & $-0,10109$ & 0,68213 & 0,42784 & 2,68246 \\
& 0,16961 & 0,23218 & $-2,53961$ & 1,91839 & $-0,65240$ & & & \\
\hline 1994 & 0,23086 & $-0,07363$ & $-0,11240$ & $-0,15770$ & $-0,04461$ & 0,52007 & 0,13613 & 1,35455 \\
& 2,04029 & $-0,47357$ & $-0,52643$ & $-0,64202$ & $-0,19055$ & & & \\
\hline & & & & & & &
\end{tabular}

1 Las cifras que figuran en las columnas segunda a sexta son las estimaciones por MCO de los parámetros de la siguiente regresión én cada año $t$ :

$$
(E / P)_{k t}=a_{0 t}+a_{t} \beta_{k r}+\sum_{i=1}^{3} b_{v} g x_{k, t+\tau}
$$

en donde: $(\mathrm{E} / \mathrm{P})_{k t}$ es el ratio $\mathrm{E} / \mathrm{P}$ mediano de la cartera $k$ al término del año $t ; \beta_{k l}$ es la mediana de las betas estimadas para cada título incluido en la cartera $k$ sobre un horizonte de hasta cinco años previos al término del año $t$; y $g x_{k, t+\tau}$ es la tasa de crecimiento mediano del resultado por acción de la cartera $k$ en el año $t+\tau$. Las cifras que aparecen debajo de las estimaciones son los valores del estadístico $t$, denotándose por * la significación de dichas estimaciones con un nivel de confianza del 95 por 100 y por ** su signifícación con un nivel de confianza del 90 por 100 (en ambos casos se emplea un test de dos colas). Esta miśma notación se utiliża para el estadístico $F$, que figura en la última columna. 
Así pues, nuestros resultados indican que ni el coeficiente beta ni el crecimiento futuro de los resultados constituyen determinantes fundamentales del ratio E/P en el mercado de valores español. Esto puede deberse a varias razones. Por una parte, puede interpretarse que los resultados contables son una buena medida de la riqueza generada por las empresas, pero que los precios alcanzados por los títulos en el mercado de valores español no son una estimación eficiente del valor de las sociedades. Esto implicaría asumir que el mercado no es eficiente y valora erróneamente a las empresas aplicándoles un múltiplo a sus resultados que no es coherente con los determinantes fundamentales de su valor. Por otra parte, puede interpretarse que el mercado se comporta de manera eficiente y que la beta estimada a partir del modelo de mercado no es una estimación apropiada del riesgo, existiendo factores de riesgo que el mercado identifica y descuenta adecuadamente en los precios. Finalmente, cabe la posibilidad de que los inversores no tengan capacidad para predecir el comportamiento futuro de los resultados. Esta interpretación es consistente con los resultados de Larrán y Rees [1999], que sugieren que los pronósticos de resultados de los analistas están sistemáticamente sesgados al alza, lo que daría lugar a valores sistemáticamente sesgados a la baja del ratio E/P, especialmente para aquellas empresas para las que exista una menor cantidad de información disponible en el mercado.

\section{CONCLUSIONES}

El ratio PER se emplea en el análisis de inversiones considerándolo como un múltiplo eficiente para la valoración de acciones, que viene determinado por las expectativas del mercado en relación con el riesgo asociado a la inversión y los beneficios futuros de la empresa.

El análisis de los datos correspondientes a una muestra de empresas admitidas a cotización en la Bolsa de Madrid durante el período 19821996 ha puesto de manifiesto que el ratio PER muestra un comportamiento altamente persistente, de modo que las diferencias observadas entre los valores del ratio para las carteras extremas se mantienen hasta diez años después de su formación. Esto podría deberse a la existencia de factores económicos tales como expectativas de crecimiento (riesgo)

puede caracterizar a las políticas contables de las empresas. Por ello, no pretendemos que esta afirmación sea interpretada como una conclusión definitiva sobre el impacto de las políticas contables de las empresas en los valores del PER. 
para ciertas empresas significativamente superiores (inferiores) a las del resto, o factores contables tales como una política sistemáticamente conservadora, que persisten en el largo plazo y justifican la desviación sistemática de los valores del PER con respecto a sus valores normales.

$\mathrm{Al}$ analizar la relación del PER con el crecimiento futuro de los resultados encontramos que, por término medio, las empresas que muestran valores extremadamente elevados (reducidos) del ratio en un año, experimentan importantes reversiones a la baja (alza) de sus resultados en los años inmediatamente siguientes. Esto puede interpretarse como una muestra de la capacidad del ratio PER para identificar empresas cuyos resultados contienen una elevada proporción de componentes transitorios.

Por último, la estimación de una regresión de los valores del ratio PER observados en cada año sobre la beta del CAPM y el crecimiento observado de los resultados en los tres años siguientes reveló que ni la primera ni los segundos tienen una significativa capacidad explicativa. Dado que al neutralizar en el denominador del ratio las diferencias en las políticas de amortización de las empresas, se alcanzaron resultados muy similares, entendemos que la conclusión de B\&M en el sentido de que las políticas contables pueden explicar las diferencias observadas en el PER, debe ser interpretada con cautela.

Estos hallazgos abren varios interrogantes a los que en el futuro deberá dar respuesta la investigación: por una parte, si la incapacidad de la beta para explicar las diferencias entre los valores del PER se debe a su inutilidad como medida del riesgo asociado a la inversión, como parece sugerir la investigación reciente [Fama y French, 1996] y, en tal caso, si existen otros factores de riesgo que permiten explicar las variaciones en el PER de las empresas [ver Lev y Sougianis, 1999]. Por otra parte, los estudios futuros pueden tratar de contrastar en el mercado español si, como sugiere $\mathrm{Za}$ rowin [1990] para el caso estadounidense, los pronósticos de resultados emitidos por los analistas (considerados como subrogado de las expectativas del mercado), permiten explicar las diferencias observadas en los valores del ratio PER. De ser así, habría que concluir que los analistas tienen una limitada capacidad para predecir los resultados, ya que los valores del PER quedarían explicados con mayor precisión por las predicciones de los resultados que por las realizaciones efectivas de éstos. El sesgo optimista que según Larrán y Rees [1999] caracteriza a los pronósticos en el mercado español puede conducir a la existencia de valores sistemáticamente sesgados al alza del ratio PER. Además, conviene dedicar esfuerzos en el futuro a la investigación de la relación exisțente entre los valores del PER y el nivel de persistencia de los resultados, así como al análisis de la relación existente entre los ratios PER y Precio-Valor Contable y la rentabilidad fi- 
nanciera brutas y anormal de las empresas. Finalmente, la investigación futura deberá tratar de esclarecer hasta qué punto es posible que existan diferencias sistemáticas en factores económicos, tales como ventajas competitivas o activos intangibles, y en factores contables, tales como el grado de conservadurismo de las políticas contables, que tengan carácter persistente y en qué medida ello puede explicar la existencia de desviaciones sistemáticas del PER con respecto a sus valores normales.

\section{BIBLIOGRAFÍA}

Albrecht, W.; Lookabill, L., y McKeown, J.. [1977]: «The times series properties of annual earnings", Joumal of Accounting Research, 15, pp. 226-244.

BALL, R. [1992], "The earnings-price anomaly», Journal of Accounting and Economics, 15, pp. 319-345.

BALL, R., y WATTS, R. [1972]: "Some time series properties of accounting income», Journal of Finance, 27, pp. 663-682.

BASU, S. [1977]: «Investment performance of common stocks in relation to their price-earnings ratios: A test of the efficient market hypothesis», Journal of Finance, 32, pp. 663-682.

BEAVER, W., y Dukes, R. [1973]: «Interperiod tax allocation and (-depreciation methods: Some empirical results», Accounting Review, 48, 3, pp. 549-559.

BEAVER, W., y MoRSE, D. [1978]: «What determines price-earnings ratios?», Financial Analysts Journal, julio-agosto, pp. 65-76.

BEAVER, W.; LAMBERT, R., y MORSE, D. [1980]: «The information content of security prices», Joumal of Accounting and Economics, 2, pp. 3-28.

BLACK, F. [1980]: "The magic in earnings: economic versus accounting earnings», Financial Analysts Joumal, noviembre-diciembre, pp. 19-24.

BROoKs, L., y BuCKMASTER, D. [1976]: «Further evidence of the time series properties of accounting income», Journal of Finance, 31, 5, pp. 1359-1373.

Craig D.; Johnson, G., y JoY, M. [1987]: «Accounting methods and P/E ratios», Financial Analysts Journal, marzo-abril, pp. 41-45.

DamodaRAN, A. [1996]: Investment valuation: Tools and techniques for determined the value of any asset. John Wiley \& sons. Nueva York.

García-Ayuso CovarSí, M. [1999]: «Una evaluación empírica de la forma funcional de los ratios PER, Dividendo-Precio y Valor Contable-Precio en el mercado de capitales Español», Revista Española de Financiación y Contabilidad, 98, enero-marzo, pp. 137-177.

Gordon, M., y SHAPIRO, E. [1956]: «Capital equipment analysis: The required rate of profit», Management Science, octubre, pp. 102-110.

Damodaran, A. [1996]: Investment Valuation, New York: John Wiley \& Sons.

FERNÁNDEZ, P. [1999]: Valoración de empresas, Gestión 2000, Barcelona. 
FAMA, E. F., y FRENCH, K. R. [1996]: "The CAPM is wanted: dead or alive», Journal of Finance, 111-138.

KOTHARI, S. P., y SLOAN, R. [1992]: "Information in prices about future earnings: Implications for earnings response coefficients", Joumal of Accounting and Economics, 15, 143-171.

LARRÁN, M., y REES, W. [1999]: "Propiedades de los pronósticos de beneficios realizados por los analistas financieros: una aplicación al caso español», Revista Española de Financiación y Contabilidad, 101, julio-septiembre, pp. 675-729.

LIPE, R. C. [1986]: "The information content in the components of earnings", Journal of Accounting Research, Suplemento, 24, pp. 37-64.

Leibowitz, M., y Kogelman, S. [1990], "Inside the P/E ratio: The franchise factor», Financial Analysts Journal, noviembre-diciembre, pp. 29-43.

Lev, B., y Sougiannis, T. [1999]: "Penetrating the Book-to-Market black box: the R\&D effect», Joumal of Business, Finance and Accounting, 26, pp. 419-449.

Litzenberger, R., y Rao, C. [1971]: «Estimates of the marginal rate of time preference and average risk aversion of investors in electric utility shares: 19601966», The Bell Joumal of Economics and Management Science, primavera, pp. 265-277.

Miller, M., y Modigliani, F. [1958]: «The cost of capital, corporation finance and the theory of investment", American Economic Review, 48, pp. 261-297.

Molodovsky, N. [1959]: "Valuation of common stocks», Financial Analysts Journal, enero-febrero, pp. 23-44.

O'DONNELL, J. [1965]: "Relations between reported earnings and stock prices in the electric utility industry», Accounting Review, 40, 1, pp. 135-143.

- [1968]: "Further observations on reported earnings and stock prices», Accounting Review, 43, 3, pp. 549-563.

Ou, J., y PENMAN, S. [1989]: «Accounting measurement, price-earnings ratio, and the informational content of security prices", Joumal of Accounting Research, 27, suplemento, pp. 111-144.

Penman, S. [1996]: «The articulation of price-earnings ratios and market-to-book ratios and the evaluation of growth", Journal of Accounting Research, 34, 2, pp. 235-259.

WATTS, R., y LEFTwich [1977]: «The time series of annual accounting earnings», Journal of Accounting Research, 15, pp. 253-271.

WATts, R., y Zimmerman, J. [1978]: «Towards a positive theory of the determination of accounting standards", Accounting Review, 53, 1, pp. 112-134.

ZarowiN, P. [1990]: "What determines earnings-price ratios: Revisited», Journal of Accounting, Auditing and Finance, 6, pp. 439-457. 